\title{
Perfect and Defective ${ }^{13} \mathrm{C}$-Furan-Derived Nanothreads from Modest-Pressure Synthesis Analyzed by ${ }^{13} \mathrm{C}$ NMR
}

Bryan Matsuura, ${ }^{1}$ Steven Huss, ${ }^{2}$ Zhaoxi Zheng, ${ }^{3}$ Shichen Yuan, ${ }^{3}$ Tao Wang, ${ }^{4,5}$ Bo Chen, ${ }^{6,7}$ John V. Badding ${ }^{2,5,8,9}$ Dirk Trauner, ${ }^{1,10,11}$ Elizabeth Elacqua, ${ }^{2,8}$ Adri C.T. van Duin, ${ }^{4}$ Vincent H. Crespi, ${ }^{2,5,8,9}$ Klaus Schmidt-Rohr ${ }^{3 *}$

${ }^{1}$ : Department of Chemistry, New York University, New York, NY 10003, USA

2: Department of Chemistry, The Pennsylvania State University, University Park, Pennsylvania 16802, USA

3: Department of Chemistry, Brandeis University, Waltham, MA 02453, USA

4. Department of Mechanical Engineering, The Pennsylvania State University, University Park, Pennsylvania 16802, USA

5: Department of Physics, The Pennsylvania State University, University Park, Pennsylvania 16802, USA

${ }^{6}$ : Donostia International Physics Center, Paseo Manuel de Lardizabal, 4. 20018 Donostia-San Sebastian, Spain

7: IKERBASQUE, Basque Foundation for Science, Plaza Euskadi 5, 48009 Bilbao, Spain

8: Materials Research Institute, The Pennsylvania State University, University Park, Pennsylvania 16802, USA

9: Department of Materials Science and Engineering, The Pennsylvania State University, University Park, Pennsylvania 16802, USA

10: Perlmutter Cancer Center, New York University School of Medicine, New York, NY 10016, USA

${ }^{11}$ : NYU Neuroscience Institute, New York University School of Medicine, New York, NY 10016, USA

†: deceased. 


\section{ABSTRACT}

The molecular structure of nanothreads produced by slow compression of ${ }^{13} \mathrm{C}_{4}$-furan was studied by advanced solid-state NMR experiments. Spectral editing showed that $>95 \%$ of carbon atoms are bonded to one hydrogen $(\mathrm{C}-\mathrm{H})$, and there are $2-4 \% \mathrm{CH}_{2}, 0.6 \% \mathrm{C}=\mathrm{O}$, and $<0.3 \% \mathrm{CH}_{3}$ groups. In addition to $7 \%$ of carbon in trapped, partially mobile furan, $18 \%$ of alkene $\mathrm{C}$ was detected. Twodimensional (2D) ${ }^{13} \mathrm{C}_{-13}{ }^{13} \mathrm{C}$ and ${ }^{1} \mathrm{H}^{-13} \mathrm{C}$ NMR clearly identified $12 \% \mathrm{C}$ in asymmetric $\mathrm{O}-\mathrm{C}=\mathrm{C}-\mathrm{C}-\mathrm{C}-$ and $24 \%$ in symmetric O-C-C=C-C- rings. While many of the former represented defects or chain ends, many of the latter appeared to be found in repeating thread segments. The $14 \%$ of alkyl carbons bonded to $\mathrm{sp}^{2}$-hybridized $\mathrm{C}$, as well as some of their neighbors, were also specifically identified in 2D NMR spectra. Around $10 \%$ of carbon atoms were found in perfect fully saturated nanothread segments arising from double $\mathrm{C}_{\alpha}-\mathrm{C}_{\beta}$ linkages formed between reacting furan rings. Previously considered straight syn threads with four different $\mathrm{C}-\mathrm{H}$ bond orientations were ruled out by CODEX NMR, which was instead consistent with anti thread segments. The observed distinctive $\mathrm{O}-\mathrm{C}-\mathrm{H}{ }^{13} \mathrm{C}$ and ${ }^{1} \mathrm{H}$ chemical shifts matched those of anti but not syn or syn/anti threads in ab initio quantum-chemical simulations. Unusually slow ${ }^{13} \mathrm{C}$ spin-exchange with sites outside the perfect threads proved a length of at least 14 bonds; the small line width of the two perfectthread signals also implied such a long, regular structure. Carbons in the perfect threads underwent slower spin-lattice relaxation than in other sites, indicating slow spin exchange and smalleramplitude motions in the perfect threads. Through partial inversion recovery, the signals of the threads were observed and analyzed selectively. These observations represent the first direct determination of the atomic-level structure of well-ordered, fully saturated nanothreads.

Table of Content graphic:

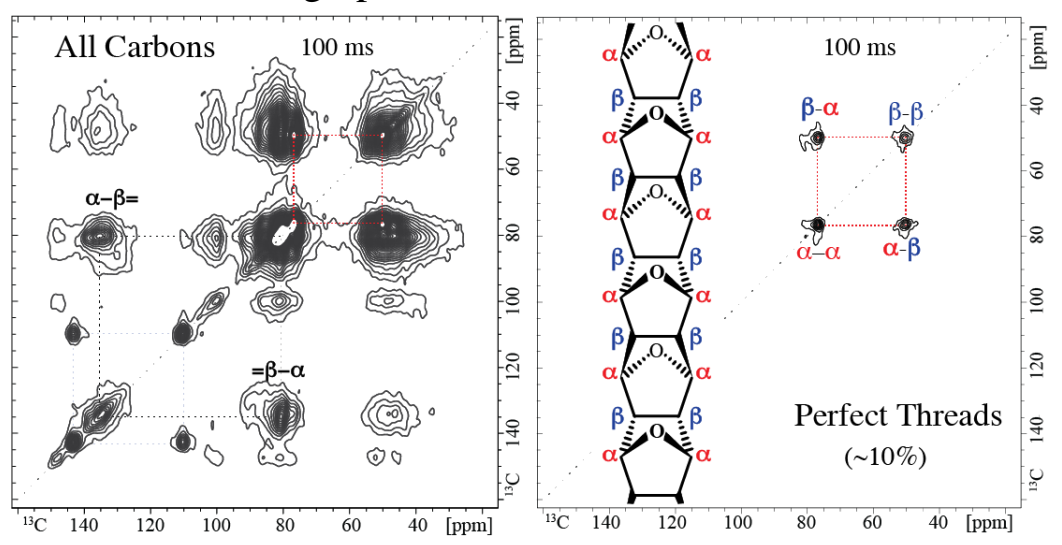




\section{INTRODUCTION}

Carbon's ability to form highly directional bonds in several different hybridizations yields a diverse panoply of molecular frameworks in zero, one, two, and three dimensions including adamantanes, ${ }^{1,2}$ fullerenes, ${ }^{3,4}$ nanotubes, ${ }^{5,6}$ nanohoops, ${ }^{7-9}$ graphene, ${ }^{10}$ graphane, ${ }^{11,}{ }^{12}$ covalent organic frameworks, ${ }^{13-15}$ and their ultimate ancestors graphite and diamond; these have garnered justifiably broad and deep interest in the scientific community. Saturated carbon nanothreads, which fill the one-dimension/ $/ \mathrm{sp}^{3}$ entry in this matrix of dimensionality and hybridization, were first synthesized by high-pressure solid-state polymerization of benzene. ${ }^{16,17}$ Since then, a wide diversity of nanothreads consisting primarily of sp $^{3}$-hybridized carbon have been synthesized by pressure-induced polymerization of aromatic molecules (e.g. pyridine, thiophene and aniline), ${ }^{18-22}$ co-crystals (e.g. naphthalene-octafluoronaphthalene, phenol-pentafluorophenol), ${ }^{23-26}$ and a strained saturated hydrocarbon, cubane. ${ }^{27}$ Pressure-induced solid-state reaction thus appears to be a general means of obtaining ordered packings of one-dimensional, high-aspect ratio diamond-like backbones decorated with diverse heteroatoms and functional groups, living at the threshold of thickness where framework rigidity first emerges in solids, a regime rife with promise for novel chemical and physical properties. ${ }^{28-31}$ Advances in our understanding of nanothread synthesis including the roles of temperature, ${ }^{19}, 21$ aromaticity, ${ }^{22}$ molecular stacking geometry, ${ }^{23-26}$ compression rate, ${ }^{16,17,22,24}$ and uniaxiality of $\operatorname{stress}^{16,17,20,22}$ - have been accompanied by substantial reductions in synthesis pressure, convincing demonstrations of $\mathrm{sp}^{3}$ character, and initial ascertainments of axial periodicity, ${ }^{32}$ but not yet by a clear determination of the precise atomic structure of regular, periodic regions of saturated thread backbone.

Mass spectrometry of furan-derived threads indicates molecular weights of about $6 \mathrm{kDa}$, consistent with 100 furan units in the backbone, ${ }^{22}$ but the detailed molecular structure has to date only been constrained by inferences as to the cross-sectional shape that follow from detailed analysis of their crystalline packing. ${ }^{22}$ Three structures based on [4+2] cycloaddition pathways have been proposed, varying in the relative placement of oxygen atoms down the nanothread axis. These threads were termed syn, anti, and syn/anti, wherein syn has eclipsed oxygens and anti has oxygen atoms alternating across the thread backbone. The intermediate syn/anti case has oxygen atoms alternating in pairs. After slow decompression from $15 \mathrm{GPa}$ to $1.5 \mathrm{GPa}$, a sharp six-fold diffraction pattern is observed in situ. Comparison of the experimentally observed $d$-spacings of Friedel pairs to those obtained from atomistic simulations of the proposed structures suggested anti and syn/anti as candidate structures, but could not distinguish between them or variants thereof. Molecularlevel information of sufficient fidelity to determine a precise nanothread structure - below the current resolution limit of electron microscopy in these systems and beyond the information so-far obtained from XRD has been lacking to date for any nanothread type. Here we report solid-state NMR measurements of isotopically enriched furan nanothreads that answer this call. 
Solid-state NMR provides unique opportunities for a comprehensive and quantitative structural analysis of complex organic materials like nanothreads on the molecular level. ${ }^{33}$ It takes advantage of structurally characteristic ${ }^{13} \mathrm{C}$ and ${ }^{1} \mathrm{H}$ chemical shifts, which are also amenable to ab initio quantum-chemical simulations. ${ }^{34}$ Unlike in vibrational spectra, peak areas in NMR are quantitative if the experiment has been performed appropriately, which means that relative concentrations of different moieties can be determined. Modern NMR involves much more than just taking "the" ${ }^{13} \mathrm{C}$ NMR spectrum. Spectral editing, e.g., in terms of the number of attached hydrogen atoms to a given carbon, ${ }^{35}$ assists in peak assignment. Two-dimensional ${ }^{1} \mathrm{H}^{13} \mathrm{C}$ spectroscopy with homonuclear ${ }^{1} \mathrm{H}$ decoupling provides access to the ${ }^{1} \mathrm{H}$ chemical shifts and with ${ }^{1} \mathrm{H}$ spin diffusion enables domain-size analysis on the 10-nm scale. Mobile segments can be identified through motional averaging of orientation-dependent spin interactions or characteristic changes in spin relaxation times. ${ }^{36}$ Materials made from ${ }^{13} \mathrm{C}$-enriched precursors provide many additional opportunities. ${ }^{33}$ The 90 -fold enrichment over the natural ${ }^{13} \mathrm{C}$ abundance of $1.1 \%$ provides a 90 fold signal enhancement that enables detection of $\mathrm{C}=\mathrm{O}, \mathrm{CH}_{3}$, and other spectrally resolved moieties at a level of $<0.1 \%$. With a ${ }^{13} \mathrm{C}$ spin in every carbon site, strong one-bond ${ }^{13} \mathrm{C}-{ }^{13} \mathrm{C}$ couplings can be exploited in two-dimensional ${ }^{13} \mathrm{C}-{ }^{13} \mathrm{C}$ NMR to determine which carbons are bonded or separated by a few bonds. ${ }^{33}$ Through multi-step ${ }^{13} \mathrm{C}$ spin exchange or spin diffusion, proximities or domains on the scale of several nanometers can be probed. In crystalline or otherwise highly ordered systems, the number of carbons in the local asymmetric unit cell (e.g. the number of differently oriented $\mathrm{C}-\mathrm{H}$ bonds in nanothreads) can be determined by CODEX NMR ${ }^{37}$ with ${ }^{13} \mathrm{C}$ spin exchange.

\section{EXPERIMENTAL}

Synthesis of ${ }^{13} \mathbf{C}_{\mathbf{4}}$-furan. As shown in Scheme 1, the synthesis of ${ }^{13} \mathrm{C}$-furan was achieved starting from commercially available ${ }^{13} \mathrm{C}_{3}$-propargyl alcohol (2) using a modified procedure adapted from $\mathrm{Vu}$ et al. ${ }^{38}$ Firstly, ${ }^{13} \mathrm{C}_{3}$-propargyl alcohol (2) was protected using the tetrahydropyranyl group under acidic conditions affording $\mathbf{3}$ in quantitative yields. Following this, $\mathbf{3}$ was deprotonated with $n$-butyl lithium and the corresponding lithium acetylide was reacted with ${ }^{13} \mathrm{C}$-labelled formaldehyde, producing 4 in $96 \%$ yield, which was subsequently deprotected to ${ }^{13} \mathrm{C}_{4}$-butyn-1,4diol (5) in moderate yield. The reported conditions for semihydrogenation of $\mathbf{5}$ were unsatisfactory in our hands and prone to over reduction and alkene isomerization. These problems were circumvented by using modified conditions, reacting 5 with 8 wt\% Lindlar's catalyst and 1.0 equivalents of quinoline in methanol under a hydrogen atmosphere, affording ${ }^{13} \mathrm{C}_{4}$-cis-buten-1,4diol (6) in $84 \%$ yield. ${ }^{39}$ Although we were able to replicate the reported conditions for the final oxidative cyclization, we found that it was unsuitable for use on small scale. After considerable experimentation using biphasic conditions ${ }^{40}$ or alternative oxidants such as Bobbitt's salt ${ }^{41}$ or 
Dess-Martin periodinane, ${ }^{42}$ we found that using substoichiometric pyridinium chlorochromate in 6:1 water/ $\mathrm{H}_{2} \mathrm{SO}_{4}$ could reliably generate ${ }^{13} \mathrm{C}_{4}$-furan (1) in $25 \%$ yield after fractional distillation. The success of this subtle modification of the reported reaction conditions is presumably due to the poor aqueous solubility of PCC, which prevented undesired over-oxidation of $\mathbf{6}$. More details are given in the SI.

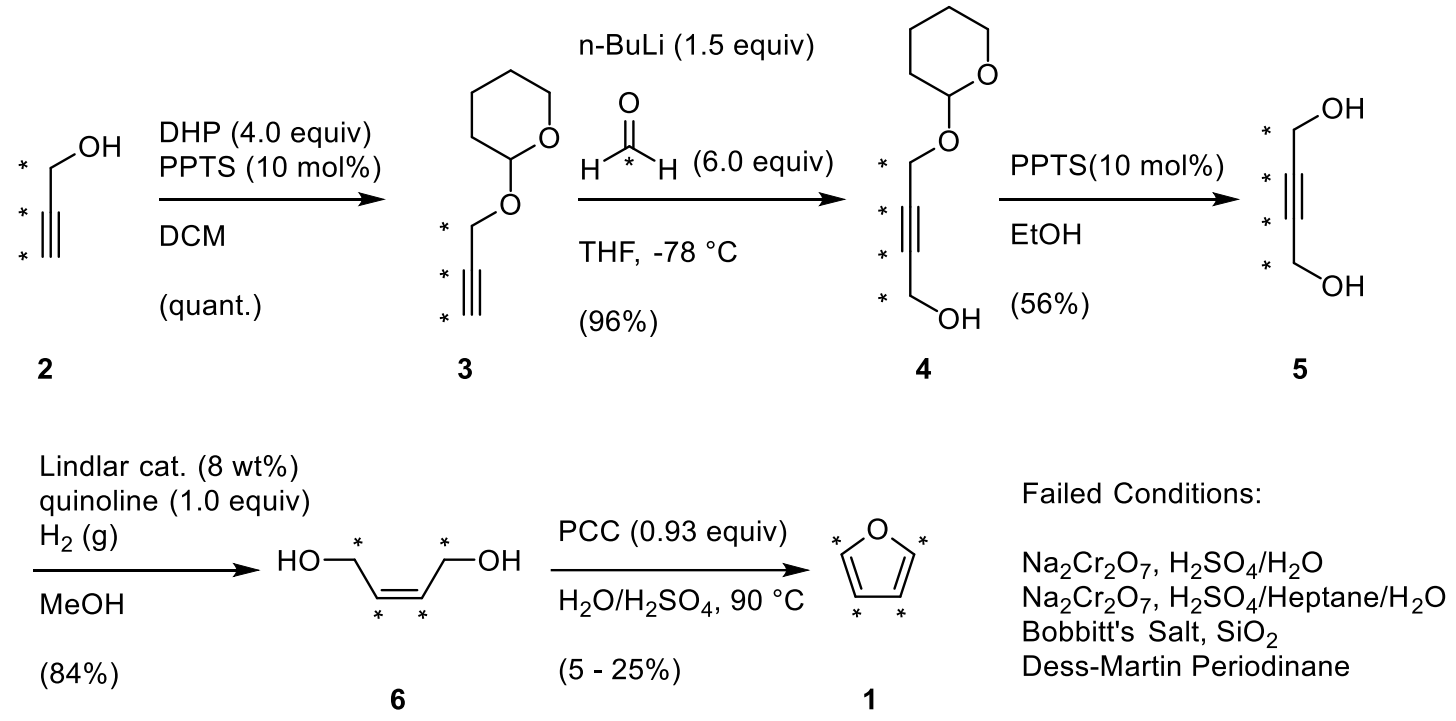

Scheme 1

High-pressure synthesis of ${ }^{13} \mathbf{C}$-furan-derived nanothreads. ${ }^{13} \mathrm{C}_{4}$-furan was loaded into an encapsulated stainless-steel gasket and slowly compressed and decompressed using a V7 ParisEdinburgh press (PE Press) equipped with double-toroid polycrystalline diamond anvils. ${ }^{43}$ Liquid nitrogen was used to freeze the liquid ${ }^{13} \mathrm{C}$-enriched furan into a solid to ensure the gasket was fully filled without any trapped air; the evaporated nitrogen gas also helped to exclude water and oxygen from the loading container. The system was driven by an automatic oil syringe pump, allowing for controllable pressure ramp rates. A pressure-load calibration curve was used from previously reported data for the double-toroid anvil design. ${ }^{44}$ The sample pressure was approximately $17 \mathrm{GPa}$ at an oil pressure of 807 bar. When the oil pressure reached 547 bar, a slow rate of increase (1 bar/min) was employed in both compression and decompression cycles. Approximately 4 milligrams of solid were produced from 21 microliters of ${ }^{13} \mathrm{C}_{4}$-furan loaded into the gasket. 
Basic solid-state NMR parameters. Solid-state NMR experiments were performed on a Bruker Avance Neo 400WB NMR spectrometer at ${ }^{1} \mathrm{H}$ and ${ }^{13} \mathrm{C}$ resonance frequencies of $400 \mathrm{MHz}$ and 100 $\mathrm{MHz}$, respectively. Most of the measurements were conducted using a Bruker double-resonance magic-angle-spinning (MAS) probe with 4-mm zirconia rotors. Approximately $4 \mathrm{mg}$ of ${ }^{13} \mathrm{C}$ furanderived nanothread sample as received was center packed into the rotor. The bottom empty space was filled by glass-fiber wool and a glass spacer, while a small Teflon cylinder was used to cap the sample. The $90^{\circ}$ pulse strengths for ${ }^{1} \mathrm{H}$ and ${ }^{13} \mathrm{C}$ were $\gamma B_{1} / 2 \pi=69 \mathrm{kHz}$ and $62 \mathrm{kHz}$, respectively. Two-pulse phase modulation (TPPM) ${ }^{45}{ }^{1} \mathrm{H}$ decoupling at a field strength of $\gamma B_{1} / 2 \pi=95 \mathrm{kHz}$ was used for ${ }^{1} \mathrm{H}$ dipolar decoupling during the Hahn echo ${ }^{46}$ or total suppression of sidebands (TOSS) ${ }^{47}$ for dead-time-free detection, while decoupling by SPINAL- $64^{48}$ at $\gamma B_{1} / 2 \pi=85 \mathrm{kHz}$ was applied during signal acquisition. ${ }^{13} \mathrm{C}$ chemical shifts were referenced externally to tetramethylsilane (TMS) using the carboxyl resonance of $\alpha-1-^{13} \mathrm{C}$-glycine at $176.49 \mathrm{ppm}$ as a secondary reference. All NMR experiments were conducted at approximately $300 \mathrm{~K}$.

An acquisition time between 6.2 and $15.5 \mathrm{~ms}$ was typically used in the one-dimensional (1D) ${ }^{13} \mathrm{C}$ NMR experiments. The ${ }^{13} \mathrm{C} B_{1}$ field strength used in cross-polarization was optimized for each MAS frequency. Unless otherwise stated, the spectra presented were acquired with recycle delays ranging from $4 \mathrm{~s}$ to $12 \mathrm{~s}$ at a MAS frequency of $14 \mathrm{kHz}$. Quantitative ${ }^{13} \mathrm{C}$ NMR spectra were measured using ${ }^{13} \mathrm{C}$ direct polarization (DP) with a recycle delay of $80 \mathrm{~s}$, averaging 576 scans were averaged for ( $21 \mathrm{~h}$ measuring time). Cross-polarization (CP) MAS ${ }^{13} \mathrm{C}$ NMR experiments were performed with a typical contact time of 1.1 ms with a 90-100 \% amplitude ramp on the ${ }^{1} \mathrm{H}$ channel. To exclude the possibility of highly crystalline furan-derived nanothreads with extremely long $T_{1 \mathrm{H}}$, a CP experiment with 3,400-s recycle delay was conducted with 32 scans being averaged over $\sim 30$ hours. The ${ }^{13} \mathrm{C}$ spin-spin relaxation time $\left(T_{2} \mathrm{C}\right)$ was measured at $14 \mathrm{kHz}$ MAS after $\mathrm{CP}$ using a Hahn spin echo ranging from $0.14 \mathrm{~ms}\left(1 \times 2 t_{\mathrm{r}}\right)$ to $3 \mathrm{~ms}\left(21 \times 2 t_{\mathrm{r}}\right)$. Peak intensities at $81 \mathrm{ppm}$ and $51 \mathrm{ppm}$ were fitted with single exponential functions with time constants of $T_{2 \mathrm{C}}=2.3 \mathrm{~s}$ and $2.2 \mathrm{~s}$, respectively, corresponding to homogeneous full widths at half maximum of $\sim 130 \mathrm{~Hz}$.

One-pulse ${ }^{1} \mathrm{H}$ NMR spectra were measured with one-pulse probehead background suppression ${ }^{49}$ at $5 \mathrm{kHz}$ with 64 scans and 15 -s recycle delays, with the ${ }^{1} \mathrm{H}$ carrier frequency set at $2.5 \mathrm{ppm} .{ }^{1} \mathrm{H}$ chemical shifts were internally referenced to highly mobile furan at 6.4 and $7.4 \mathrm{ppm}$.

Spectral editing ${ }^{13} \mathrm{C}$ NMR. Selective spectra of non-protonated ${ }^{13} \mathrm{C}$ or segments undergoing fast large-amplitude motions were recorded after direct polarization with 80-s recycle delays, using recoupled ${ }^{1} \mathrm{H}-{ }^{13} \mathrm{C}$ dipolar dephasing, with ${ }^{1} \mathrm{H}$ decoupling switched off for $40 \mu$ s and $27 \mu$ s before and after the echo $\pi$-pulse, respectively; compared to the conventional symmetric $2 \times 30 \mu$ s gated decoupling, the residual signal was reduced by a factor of 0.7 . A CH-only spectrum were obtained 
by dipolar distortionless enhancement by polarization transfer (dipolar DEPT) at $5787 \mathrm{~Hz} \mathrm{MAS.}{ }^{35}$ 4096 scans were averaged for $\sim 5$ hours. The $\mathrm{CH}_{2}$-only spectrum were obtained by three-spin coherence selection $^{50}$ at $5787 \mathrm{~Hz}$ MAS with a CP contact time of $70 \mu$ s and carefully tuned flipback pulse. 16384 scans were averaged for $\sim 23$ hours. Hydroxyl-proton selected (HOPS) ${ }^{13} \mathrm{C}$ NMR ${ }^{51}$ was performed to look for $\mathrm{C}-\mathrm{OH}$ moieties in furan-derived nanothreads. The ${ }^{1} \mathrm{H}$ on-resonance frequency was set at $10.5 \mathrm{ppm}$ for HOPS with a CP contact time of $0.25 \mathrm{~ms} .1024$ transients for both $S$ and $S_{0}$ spectra were averaged within a total time of $3 \mathrm{~h}$.

Spectra near the zero-crossing during ${ }^{13} \mathrm{C}$ inversion-recovery ${ }^{52}$ were recorded after $\mathrm{CP}$ to selectively observe components with different ${ }^{13} \mathrm{C}$ spin-lattice relaxation times $\left(T_{1 \mathrm{C}}\right)$. The pulse length of the inversion pulse after $\mathrm{CP}$ was reduced to $3 \mu$ s for less complete magnetization inversion resulting in an earlier zero-crossing of the recovering magnetization. Recovery times differing by $2 \mathrm{~s}$ were used for selective polarization of the perfect thread signals and non-perfect thread signals, respectively. For ease of illustration, the signal in some spectra using the inversionrecovery filter is shown inverted to display the negative, slowly relaxing peaks as positive. For a standard ${ }^{13} \mathrm{C}$ inversion recovery experiment, 2816 scans were averaged over $12 \mathrm{~h}$.

2D and exchange ${ }^{13} \mathrm{C}$ NMR experiments. A two-dimensional (2D) double-quantum/singlequantum (DQ/SQ, solid-state INADEQUATE) ${ }^{13} \mathrm{C}$ NMR spectrum was measured at $14 \mathrm{kHz}$ MAS using the SPC $5{ }^{53}{ }^{13} \mathrm{C}-{ }^{13} \mathrm{C}$ dipolar recoupling sequence without ${ }^{1} \mathrm{H}$ irradiation for duration of $2 \times 0.29$ $\mathrm{ms}$, relying on ${ }^{13} \mathrm{C}$ irradiation at $\left|\gamma B_{1}\right| / 2 \pi=70 \mathrm{kHz}$ for heteronuclear decoupling. The total acquisition time was $69 \mathrm{~h}$. Shearing of the DQ/SQ spectrum ${ }^{54}$ to match the appearance of 2D exchange spectra was performed via the "ptilt1" functionality in TopSpin 4.0.4 using alpha1= alpha2 $=0.5$.

Two-dimensional ${ }^{1} \mathrm{H}^{13} \mathrm{C}$ heteronuclear correlation (HetCor) ${ }^{55}$ spectra were measured at a $7.5 \mathrm{kHz}$ MAS frequency with frequency-switched Lee-Goldburg homonuclear ${ }^{1} \mathrm{H}$ decoupling at a pulse strength of $85 \mathrm{kHz},{ }^{56}$ and TOSS before detection. ${ }^{1} \mathrm{H}$ spin-diffusion was allowed to occur during a mixing time ranging from $10 \mu \mathrm{s}$ to $10 \mathrm{~ms}$. For longer mixing times ( 3 and $10 \mathrm{~ms}$ ), a CP contact time of $500 \mu$ s was used, otherwise the CP contact time was $70 \mu \mathrm{s}$. A typical spectrum was signalaveraged for 17 to 21 hours ( $93 \mathrm{~h}$ total). The ACD/NMR predictor ${ }^{57}$ was used to predict ${ }^{1} \mathrm{H}$ and ${ }^{13} \mathrm{C}$ chemical shifts in alkene-containing structures.

Two-dimensional ${ }^{13} \mathrm{C}-{ }^{13} \mathrm{C}$ exchange spectra were recorded at $14 \mathrm{kHz}$ MAS with mixing times ranging from $10 \mathrm{~ms}$ to $1 \mathrm{~s}$. For the experiment with a mixing time of $10 \mathrm{~ms}$, dipolar assisted rotational resonance (DARR) by weak ${ }^{1} \mathrm{H}$ irradiation ${ }^{58}$ was used to promote ${ }^{13} \mathrm{C}-{ }^{13} \mathrm{C}$ spinexchange. The measurement time per $2 \mathrm{D}$ spectrum was 12 to $21 \mathrm{~h}$. For selective observation of 
spin exchange among perfect-thread carbons, a mixing time of $100 \mathrm{~ms}$ was used in a $2 \mathrm{D}{ }^{13} \mathrm{C}-{ }^{13} \mathrm{C}$ exchange spectrum after a $6 \mathrm{~s}{ }^{13} \mathrm{C}$ inversion recovery filter (27 hours measurement time).

${ }^{13} \mathrm{C}$ spin exchange out of the perfect thread segments was observed after $6.7 \mathrm{~s}$ inversion recovery that suppresses the signals of the other threads, followed by a $36 \mu$ s chemical shift filter to suppress the total integral of the furan signals at $110 \mathrm{ppm}$ and $143 \mathrm{ppm}$, with the ${ }^{13} \mathrm{C}$ carrier frequency set to $65 \mathrm{ppm}$. The chemical-shift filter was followed by ${ }^{13} \mathrm{C}$ spin-diffusion (ranging from $3 \mathrm{~ms}$ to $3 \mathrm{~s}$ ) before detection. 512 transients for each mixing time were averaged for $\sim 15$ hours.

Centerband-only detection of exchange (CODEX) NMR experiments ${ }^{37}$ for a series of mixing times were performed at a $14 \mathrm{kHz}$ MAS frequency. It was confirmed experimentally that $N t_{\mathrm{r}}=1.14 \mathrm{~ms}$ (with $2 \times 15 \pi$-pulses) produced a well-dephased $S$ spectrum in the long-time limit. The ${ }^{13} \mathrm{C}-{ }^{13} \mathrm{C}$ spin-exchange/diffusion along the threads was probed using 10 mixing times ranging from $2 \mathrm{~ms}$ to $1 \mathrm{~s}$. The total experimental time for all the CODEX experiments was $\sim 20$ hours. An 8.5 -s ${ }^{13} \mathrm{C}$ inversion recovery filter was applied before the CODEX evolution period to selectively probe the spin-diffusion behavior of the perfect threads, for four mixing times, requiring a total of $\sim 7$ days of signal averaging. Spin exchange dynamics were simulated in MATLAB.

Quantum-chemical simulations. The NMR chemical shielding tensors of carbon in furan syn, anti, syn/anti, [2+2] polymer and 1,3-polymer structures were calculated in the framework of density functional theory with the gauge-including projector augmented wave (GIPAW) method ${ }^{59-}$ 62 implemented in Quantum ESPRESSO. ${ }^{63}$ The GIPAW reconstructed pseudopotentials ${ }^{64}$ with the Perdew-Burke-Ernzerhof (PBE) ${ }^{65}$ functional using the Trouillier-Martins norm-conserving method was used for all calculations, with 100 Ry energy cutoff and $<0.24 \AA^{-1} \mathrm{k}$-point spacing to obtain converged NMR parameters with reasonable computational cost. The isotropic chemical shift was calculated as ${ }^{66} 67$ :

$$
\delta_{\text {iso }}^{\text {calc }}=\frac{\sigma_{\text {iso }}^{\text {ref }}-\sigma_{\text {iso }}^{\text {calc }}}{1-\sigma_{\text {iso }}^{\text {ref }}}
$$

where $\sigma_{i s o}^{\text {calc }}$ is the calculated isotropic chemical shielding and $\sigma_{i s o}^{r e f}$ is the reference isotropic chemical shielding. To minimize systematic errors, $\sigma_{i s o}^{r e f}$ was determined by linear fitting of the calculated isotropic chemical shielding values for several structurally related systems to their known experimental isotropic chemical shifts with the equation $-\sigma_{\text {iso }}^{\text {calc }}=m \delta_{\text {iso }}^{\text {expt }}-\sigma_{i s o}^{r e f}{ }^{68}$

\section{RESULTS}


Quantitative ${ }^{13} \mathbf{C}$ NMR. Figure 1a shows a quantitative, fully relaxed direct-polarization ${ }^{13} \mathrm{C}$ NMR spectrum of ${ }^{13} \mathrm{C}$-furan-derived nanothreads. It exhibits eight peak maxima and several shoulders. The structural moieties associated with these spectral features will be identified in the following through spectral editing, ${ }^{1} \mathrm{H}-{ }^{13} \mathrm{C}$, and, most importantly, two-dimensional ${ }^{13} \mathrm{C}-{ }^{13} \mathrm{C}$ NMR. Peak areas (also taking into account spinning sidebands, which do not overlap with centerbands here) are quantitative. It is found that $25 \%$ of carbons resonate at $\geq 100 \mathrm{ppm}$, which means that they are $\mathrm{sp}^{2}$-hybridized. The intensity of the alkyl $\beta$-carbons ( $\mathrm{C}$ not bonded to $\left.\mathrm{O}\right)$ is significantly lower than that of the $\alpha$-carbon $(\mathrm{OCH})$ peak. This initially unexpected asymmetry will be fully explained below in terms of a significant fraction of alkene $\beta$-carbons.

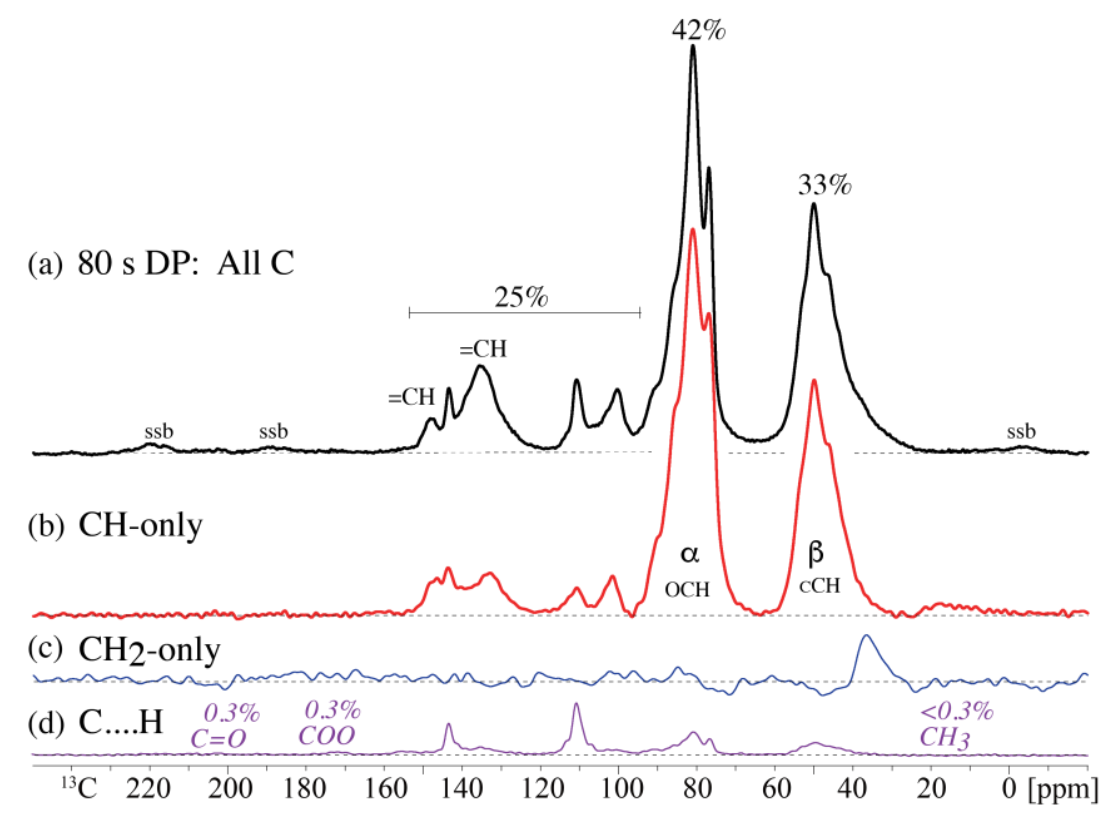

Figure 1. ${ }^{13} \mathrm{C}$ NMR of ${ }^{13} \mathrm{C}$-furan-derived nanothreads with spectral editing. (a) Quantitative directpolarization ${ }^{13} \mathrm{C}$ NMR spectrum. (b) $\mathrm{CH}$-only spectrum at $5.787 \mathrm{kHz}$ MAS. (c) $\mathrm{CH}_{2}$-only spectrum. (d) Direct-polarization spectrum after recoupled dipolar dephasing, showing mobile furan but little signal of $\mathrm{C}$ not bonded to $\mathrm{H}$ or of $\mathrm{CH}_{3}$ groups.

Spectral editing in ${ }^{13} \mathrm{C}$ NMR. A selective spectrum of $\mathrm{CH}$ groups (carbon bonded to one hydrogen), obtained by dipolar DEPT, is shown in Figure 1b. Most peaks are retained, as expected in furan-derived nanothreads. $\mathrm{A} \mathrm{CH}_{2}$-only spectrum obtained by three-spin coherence selection accordingly shows only one peak, near $38 \mathrm{ppm}$; the corresponding foot in the full spectrum represents $2-4 \%$ of the total intensity. The spectrum of carbons with weak dipolar couplings to $\mathrm{H}$, obtained by recoupled dipolar dephasing and shown in Figure 1d, exhibits little signal. The two sharp peaks are at the resonance frequency of furan and can be assigned to trapped furan molecules 
with anisotropic mobility. With sufficient vertical expansion, $\mathrm{COO}$ and ketone bands of $0.3 \%$ signal fraction each can be recognized, see Figure S1. In total, the data show that $>95 \%$ of all carbons are bonded to one hydrogen, which is characteristic of nanothreads made from unsubstituted single aromatic rings. Due to an apparent $\mathrm{OH}$ band in the IR spectrum, ${ }^{22,69}$ we searched for $\mathrm{C}-\mathrm{OH}$ signals using hydroxyl-proton selection (HOPS) $\mathrm{NMR},{ }^{51}$ but no such signals were recognized, see Figure S2, above the detection limit of $\sim 2 \%$.
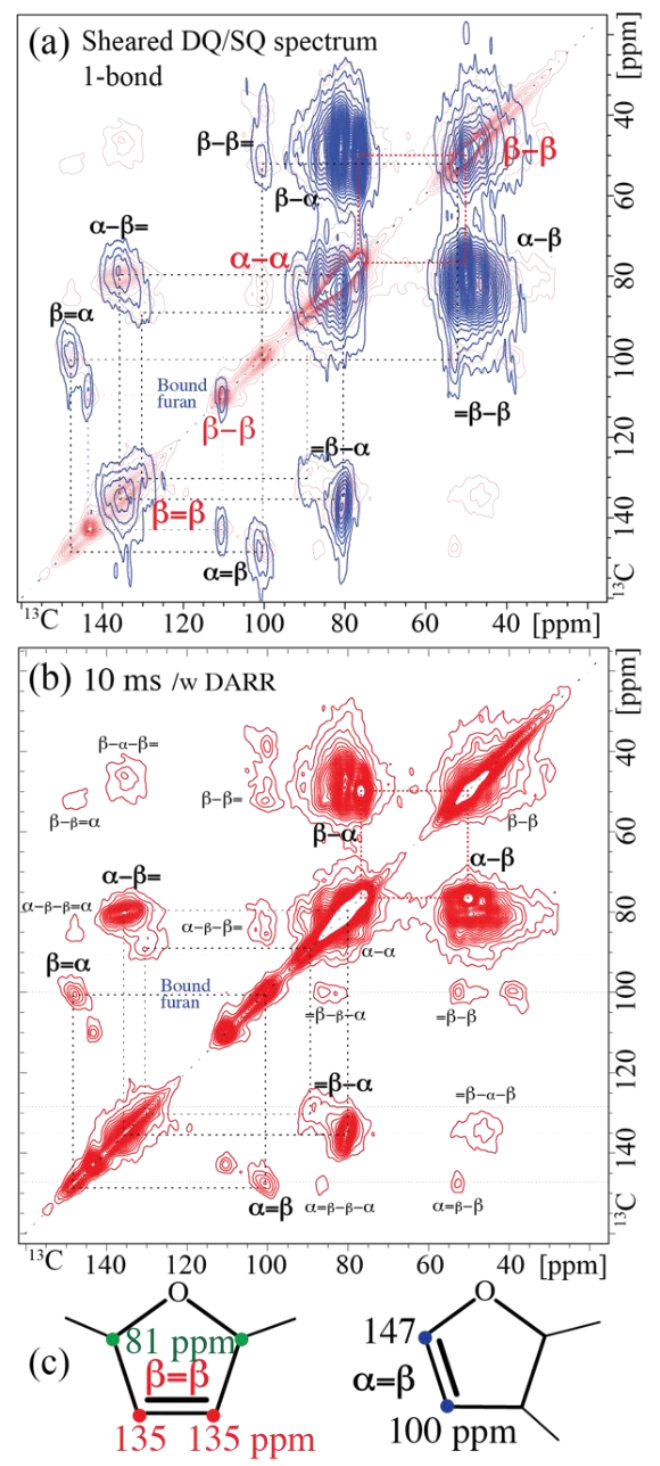

Figure 2. Two-dimensional ${ }^{13} \mathrm{C}-{ }^{13} \mathrm{C}$ NMR of ${ }^{13} \mathrm{C}$-furan-derived nanothreads. (a) Sheared DQ/SQ spectrum. (b) Exchange spectrum with a $10 \mathrm{~ms}$ mixing time and application of weak ${ }^{1} \mathrm{H}$ irradiation for dipolar assisted rotational resonance (DARR). This spectrum is also shown faintly in the background in a). (c) Symmetric and asymmetric alkene-containing rings proven by diagonal peaks (or their absence) in a) and cross peaks in a) and b). 
One-bond 2D NMR: Alkene identification. The $=\mathrm{C}-\mathrm{H}$ signals between 100 and $150 \mathrm{ppm}$ in the ${ }^{13} \mathrm{C}$ spectrum can be assigned based on characteristic cross peaks in $2 \mathrm{D}^{13} \mathrm{C}-{ }^{13} \mathrm{C}$ and ${ }^{1} \mathrm{H}-{ }^{13} \mathrm{C}$ NMR spectra with mostly one-bond transfer. Figure 2 shows ${ }^{13} \mathrm{C}-{ }^{13} \mathrm{C}$ spectra with one- and weak twobond correlation peaks. Figure $2 \mathrm{a}$ is a $\mathrm{DQ} / \mathrm{SQ}$ correlation spectrum analogous to an INADEQUATE spectrum in solution NMR but sheared to take the appearance of an exchange NMR spectrum with cross peaks ${ }^{70}$ matching the spectrum in Figure $2 \mathrm{~b}$. While the cross peaks in (a) are broader than in (b), diagonal peaks in (a) are highly valuable in showing that two carbons with very similar chemical shifts are bonded to each other (often indicating a symmetric structure), while the exchange spectrum in (b) contains meaningless diagonal peaks. Such signals of chemically equivalent spins are not observable in J-coupling-based INADEQUATE but are generated by the recoupled ${ }^{13} \mathrm{C}-{ }^{13} \mathrm{C}$ dipolar coupling in the solid state. For instance, two carbons with chemical shifts near $135 \mathrm{ppm}$ are bonded to each other; in conjunction with the (135 ppm, 81 ppm) cross peak prominent in both Figures $2 \mathrm{a}$ and $\mathrm{b}$, this proves a symmetric ring structure with a $\beta=\beta$ double bond, see Figure $2 \mathrm{c}$. The directly bonded $\beta$-carbons of the trapped furan also give rise to a clear diagonal peak near $111 \mathrm{ppm}$. Strong cross peaks between 147 and $100 \mathrm{ppm}$ prove an O$\mathrm{CH}=\mathrm{CH}$ - fragment, see Figure 2c; since it is in an asymmetric ring structure (after all, the symmetric structure would be a furan ring), it does not have associated diagonal peaks in Figure 2a.

These alkene assignments can be confirmed in a $2 \mathrm{D}{ }^{1} \mathrm{H}-{ }^{13} \mathrm{C}$ NMR spectrum with short, one-bond cross polarization from ${ }^{1} \mathrm{H}$ to ${ }^{13} \mathrm{C}$, see Figure $\mathrm{S} 3(\mathrm{a})$, where the ${ }^{1} \mathrm{H}$ chemical shifts of the $\mathrm{C}=\mathrm{C}-\mathrm{H}$ units can be read off. Characteristic $\mathrm{C}=\mathrm{C}-{ }^{1} \mathrm{H}$ chemical shifts of $6.1 \mathrm{ppm}$ and $4.9 \mathrm{ppm}$ are observed. The agreement with chemical shifts from empirical predictions using the ACD/NMR Predictors is good, see Figure S3(b).

Alkyls near alkenes. By extending the mixing time in spin-exchange $2 \mathrm{D}^{13} \mathrm{C}-{ }^{13} \mathrm{C}$ NMR to $100 \mathrm{~ms}$, we can probe proximities over several bonds. The 100-ms exchange spectrum shown in Figure 3a has stronger long-range exchange peaks compared to the few-bond-transfer spectra in Figure 2. The peak positions can be assessed most conveniently in horizontal cross sections through the $2 \mathrm{D}$ spectrum. These are equivalent to spectra obtained by selective excitation at the evolution frequency $\omega_{1}$ (the position of the peak on the diagonal) followed by spin exchange during the mixing time, e.g. $100 \mathrm{~ms}$ here. ${ }^{71}$

Selected horizontal cross sections through the 10-ms and 100-ms spectra (in blue and red, respectively) are shown in Figure 3b, with the diagonal-peak intensity matched for clarity. The biggest secondary peaks in the 10-ms spectrum are due to the one-bond couplings already discussed. Two- and three bond transfer peaks are much smaller in amplitude but are usually significantly enhanced after $100 \mathrm{~ms}$ of spin exchange. Some of the chemical shift assignments 
deduced are shown in the structures next to the spectra. The cross peaks from alkenes to $\mathrm{OCH}$ at 89,86 , and $81 \mathrm{ppm}$ can account for shoulders and peaks in the complex OCH resonance, as indicated schematically in the 1D spectrum above the 2D spectrum in Figure 3a.
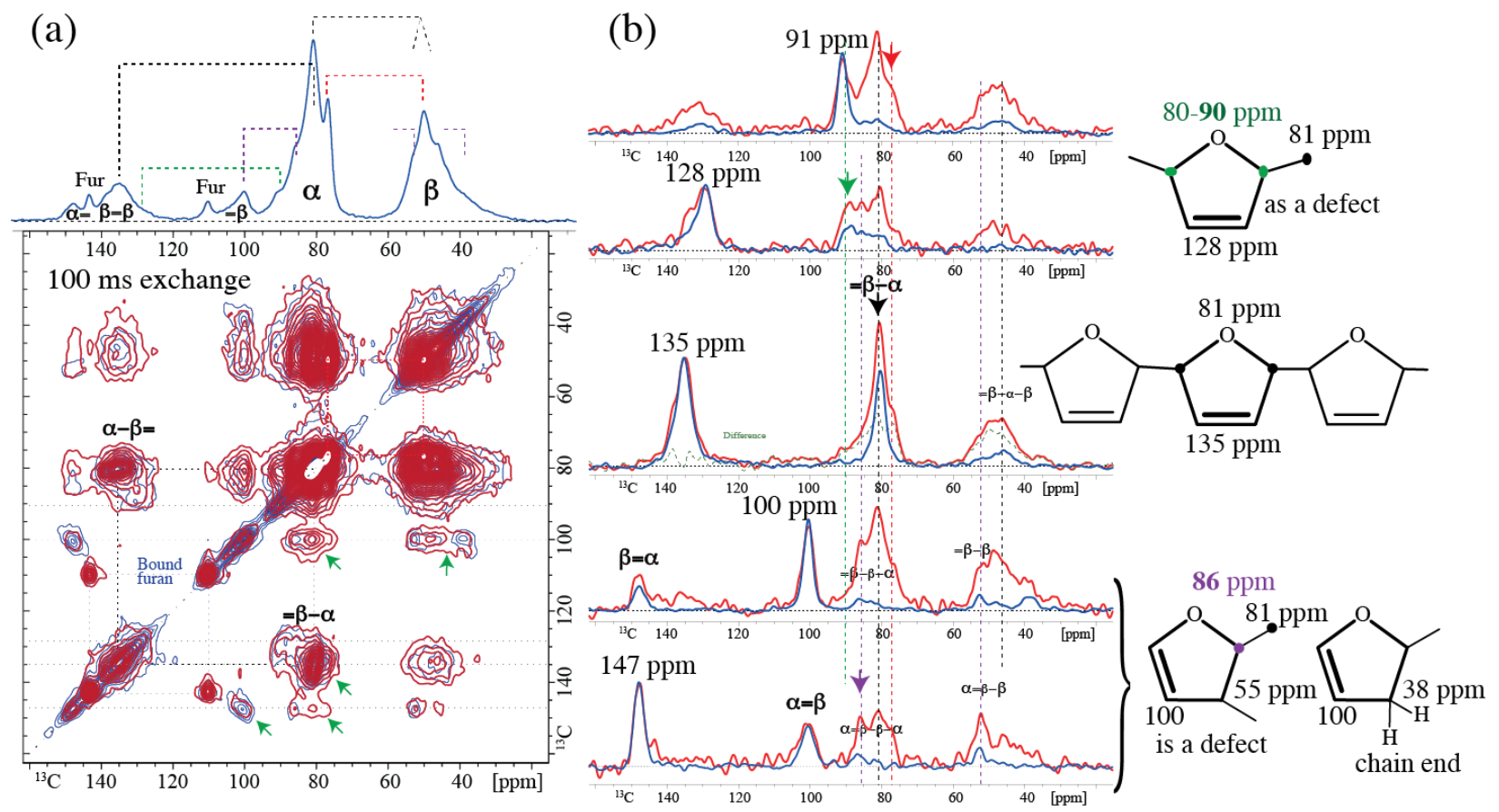

Figure 3. Few-bond connectivity of ${ }^{13} \mathrm{C}$-furan-derived nanothreads. (a) $2 \mathrm{D}$ exchange spectra with $100 \mathrm{~ms}$ mixing time (red) and $100 \mathrm{~ms}$ (thin blue) overlayed, and (b) horizontal cross sections from the spectra in (a), with the intensities of the diagonal peaks matched. In the top-left spectrum, assignments of peaks and shoulders of the $\mathrm{OCH}$ peak are indicated schematically.

Perfect-thread signals in $2 \mathrm{D}^{13} \mathrm{C}-{ }^{13} \mathrm{C}$ NMR spectra. The analysis so far has mostly focused on the relatively low peaks associated with the various types of alkenes. Interesting features also become apparent in the alkyl-alkyl correlation region, when the 100-ms 2D exchange spectrum, processed with minimum digital line broadening, is plotted at much higher contour levels, see Figure 4a. The graph reveals an intriguing set of four sharp peaks arranged in a square, superimposed on broader and mostly lower signals. The four sharp peaks of similar intensity are indicative of exchange within a highly symmetric and repetitive environment with just two chemically inequivalent sites, $\mathrm{CH}$ and $\mathrm{OCH}$ with chemical shifts of $50 \mathrm{ppm}$ and $77 \mathrm{ppm}$, respectively. 


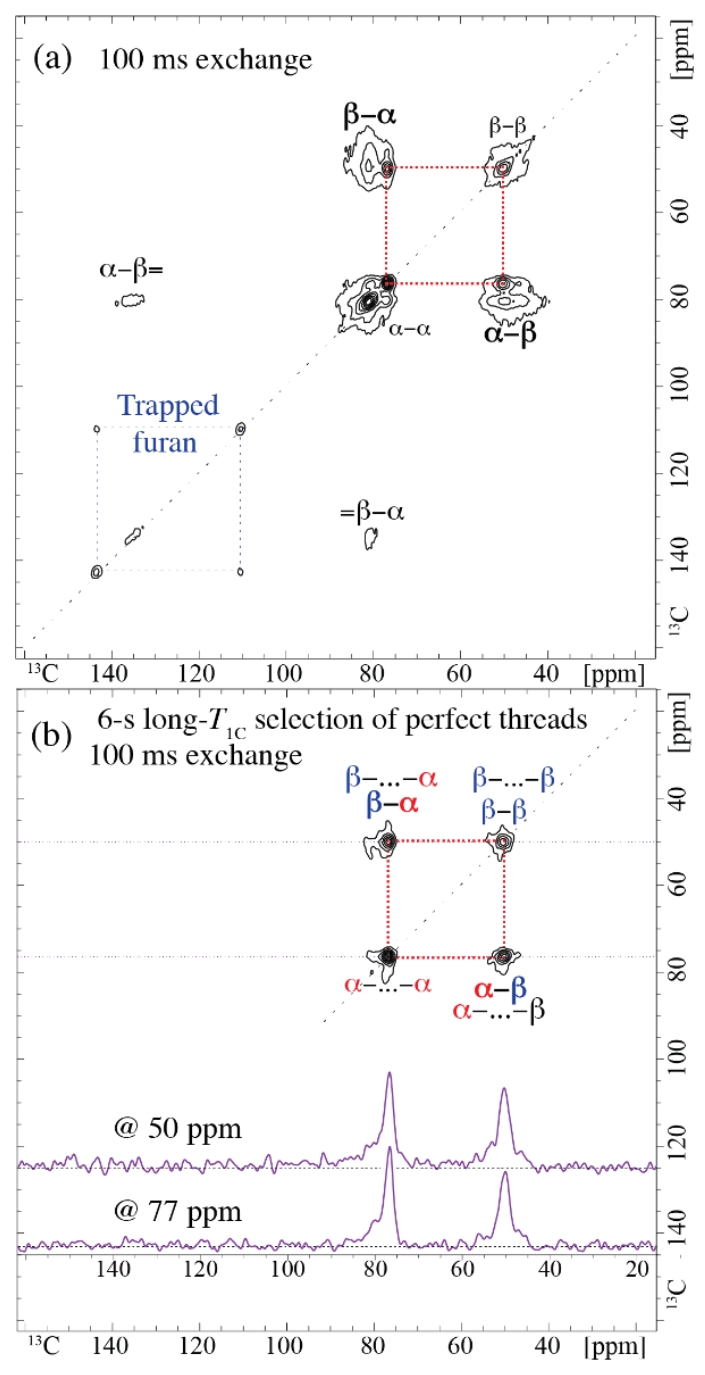

Figure 4. Two-dimensional ${ }^{13} \mathrm{C}-{ }^{13} \mathrm{C}$ NMR spectra of ${ }^{13} \mathrm{C}$-furan-derived nanothreads, highlighting signals of perfect threads. (a) Exchange NMR with 100 ms mixing time (same data as in Figure 3a, but without digital smoothing and plotted at higher contour levels). (b) after selection of the longest- $T_{1 \mathrm{C}}$ component by inversion-recovery. Horizontal cross sections along the dashed lines are also shown.

Based on their slower inversion recovery, described in the following, the $2 \mathrm{D}$ spectrum of the perfect threads can be observed selectively, see Figure 4b. Cross sections at the 50 and $77 \mathrm{ppm}$ frequencies (also in Figure 4b) show the two peaks in a 1:1 ratio, as expected for fully saturated furan-derived nanothreads, without much cross-talk to other carbon sites.

The bonding in the perfect threads can be determined by analyzing their signals in the sheared DQ/SQ spectrum as plotted in Figure S4. Strong $C_{\alpha}-C_{\beta}$ cross peaks document predominant bond formation between $\mathrm{C}_{\alpha}$ of one ring and $\mathrm{C}_{\beta}$ of a neighbor. While a $\mathrm{C}_{\beta}-\mathrm{C}_{\beta}$ diagonal peak is also clearly 
observed and expected as a result for the $\mathrm{C}_{\beta}-\mathrm{C}_{\beta}$ bond in furan, no significant $\mathrm{C}_{\alpha}-\mathrm{C}_{\alpha}$ diagonal peak is observed, indicating the absence of $\mathrm{C}_{\alpha}-\mathrm{C}_{\alpha}$ bonding in the perfect threads.

The perfect threads also stand out in an exchange spectrum with an even longer mixing time of 1000 ms, see Figure S5. All other nanothread sites reach spin-exchange equilibrium: regardless of their initial frequency along the vertical axis, the magnetization distributes over all types of carbon in their representative proportions, which means that an equilibrated horizontal cross section is a scaled version of the unselective one-dimensional spectrum. This is the case at all frequencies except 50 and 77 ppm, where the peaks of the perfect threads remain enhanced because they have not shared most of their magnetization with other carbon sites.

Slower inversion recovery of perfect-thread signals. Selection of the perfect-thread signals is possible based on their relatively slow spin-lattice relaxation. While the standard methods of $T_{1 \mathrm{C}^{-}}$ selective spectroscopy, direct polarization with short recycle delay or phase-cycled z-filtering, were not discriminatory enough to cleanly separate the signals, the selection was achieved by inversion recovery timed such that the signal of the faster relaxing defective threads just passes through zero while that of the slower-relaxing perfect threads remains significantly inverted, see Figure 5. The signals of mobile furan, which relaxes faster, have already passed through zero as they approach thermal equilibrium.

The observation that the sharp signals of the perfect threads relax the most slowly has at least two implications. First, it indicates a particularly long intrinsic $T_{1 \mathrm{C}}$ relaxation time of the perfect threads, which in turn implies particularly limited molecular motion or less contact with fastrelaxing mobile furan. Spin-lattice relaxation is driven by fluctuating magnetic fields, e.g. due to the orientation-dependent ${ }^{13} \mathrm{C}-{ }^{1} \mathrm{H}$ dipolar couplings made time-dependent by molecular motions, specifically those with spectral density at the nuclear Larmor frequency $(2 \pi 100 \mathrm{MHz}$ here, corresponding to correlation times of a few nanoseconds). The decreased relaxation rate in the perfect threads is mostly likely due to a reduced motional amplitude, consistent with an ordered, ladder-type structure with little torsional flexibility.

The second implication is a significant length of a periodic structure containing only 50 and 77 ppm sites in a 1:1 ratio. During the multi-second recovery period after inversion, multistep ${ }^{13} \mathrm{C}$ spin exchange occurs, as shown in Figure S8a,b and discussed in more detail below. If the segments containing the 50 and 77 ppm carbon sites were dispersed among the other alkyl and the alkenecontaining structures, ${ }^{13} \mathrm{C}-{ }^{13} \mathrm{C}$ spin exchange would erase the effect of different intrinsic spinlattice relaxation times and all segments would relax with the same time constant. The slow relaxation in perfect threads raises the possibility that perfect threads in large crystallites might 
relax so slowly that their signal has only minimally recovered in the recycle delays of $6-80 \mathrm{~s}$ used in most experiments. This hypothesis was ruled out by cross polarization ${ }^{13} \mathrm{C}$ NMR with 3,400 s recycle delay, which showed no additional alkyl carbon signal, see Figure S6.

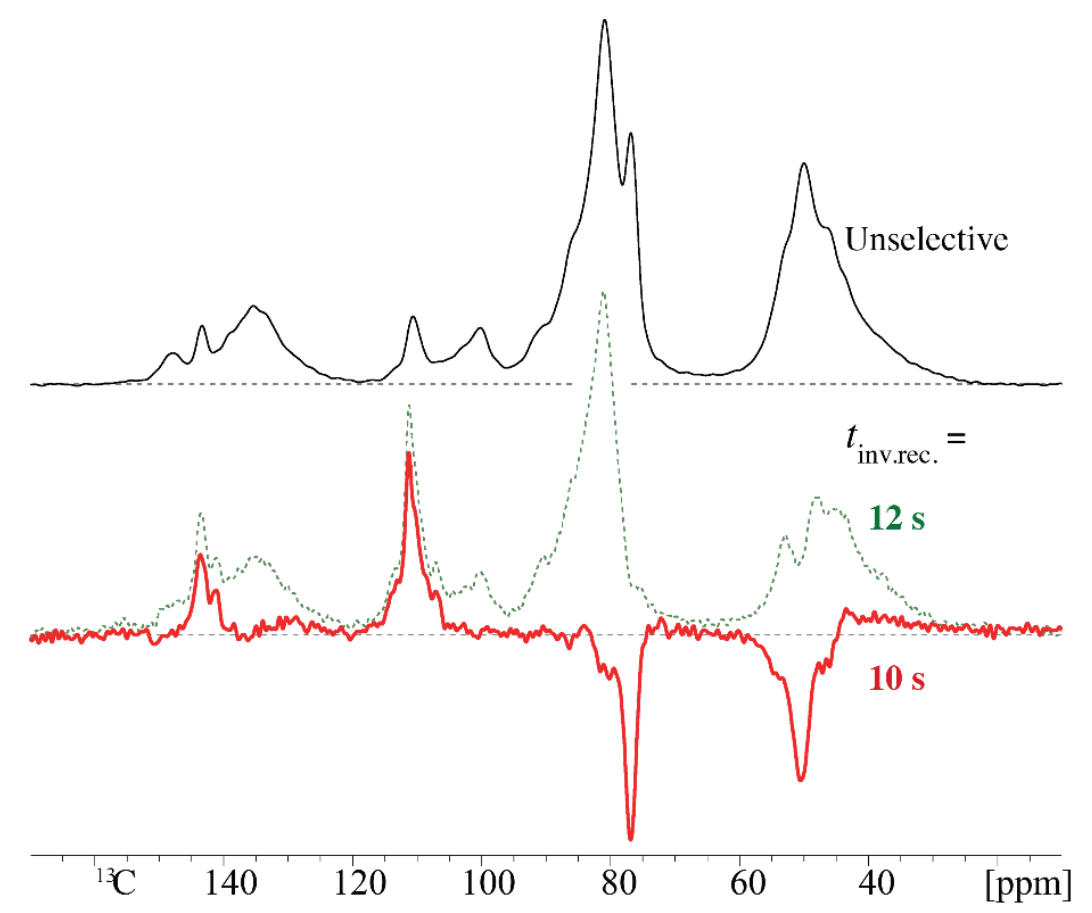

Figure 5. Spectra during ${ }^{13} \mathrm{C}$ inversion recovery in ${ }^{13} \mathrm{C}$-furan-derived nanothreads, after (bottom red trace) $10 \mathrm{~s}$ and (middle dashed trace) $12 \mathrm{~s}$ of recovery time. An unselective DP spectrum, scaled to the same height of the 81-ppm peak, is shown at the top for reference.

Spin exchange after inversion recovery. After inversion recovery to the zero crossing of the defective-thread signals, the spatial distribution of ${ }^{13} \mathrm{C}$ magnetization is in a state far from equilibrium. It will therefore undergo further ${ }^{13} \mathrm{C}$ spin exchange during a subsequent $\pm \mathrm{z}$-filtered mixing time, with the spectrum slowly approaching the regular shape observed without selective excitation. The rate of decrease of the selected peaks is indicative of the length of the perfect thread segments. The total area under the signal is initially constant and then decreases exponentially towards zero as the mixing time increases, with the $T_{1 \mathrm{C}}$ relaxation time as the time constant. The perfect-thread signal intensities can be corrected for the moderate reduction in overall signal.

In the selective spectrum of the perfect threads in Figure 5 (bottom), in addition to the desired negative signals of the slowly relaxing 77- and 51-ppm peaks, positive peaks of mobile furan are observed. These can be reduced in intensity, specifically their combined overall intensity, by a simple fixed evolution period of 36 us between inversion recovery and additional spin exchange, which acts as a chemical shift filter. The resulting sinusoidal excitation function is indicated dashed in Figure 6a. The furan peaks are reduced in intensity and have opposite signs, so the net 
magnetization in furan is very small and will not interfere with the spin exchange out of the perfect thread segments. The pulse sequence with all the mentioned elements is shown in Figure S7.

(a)
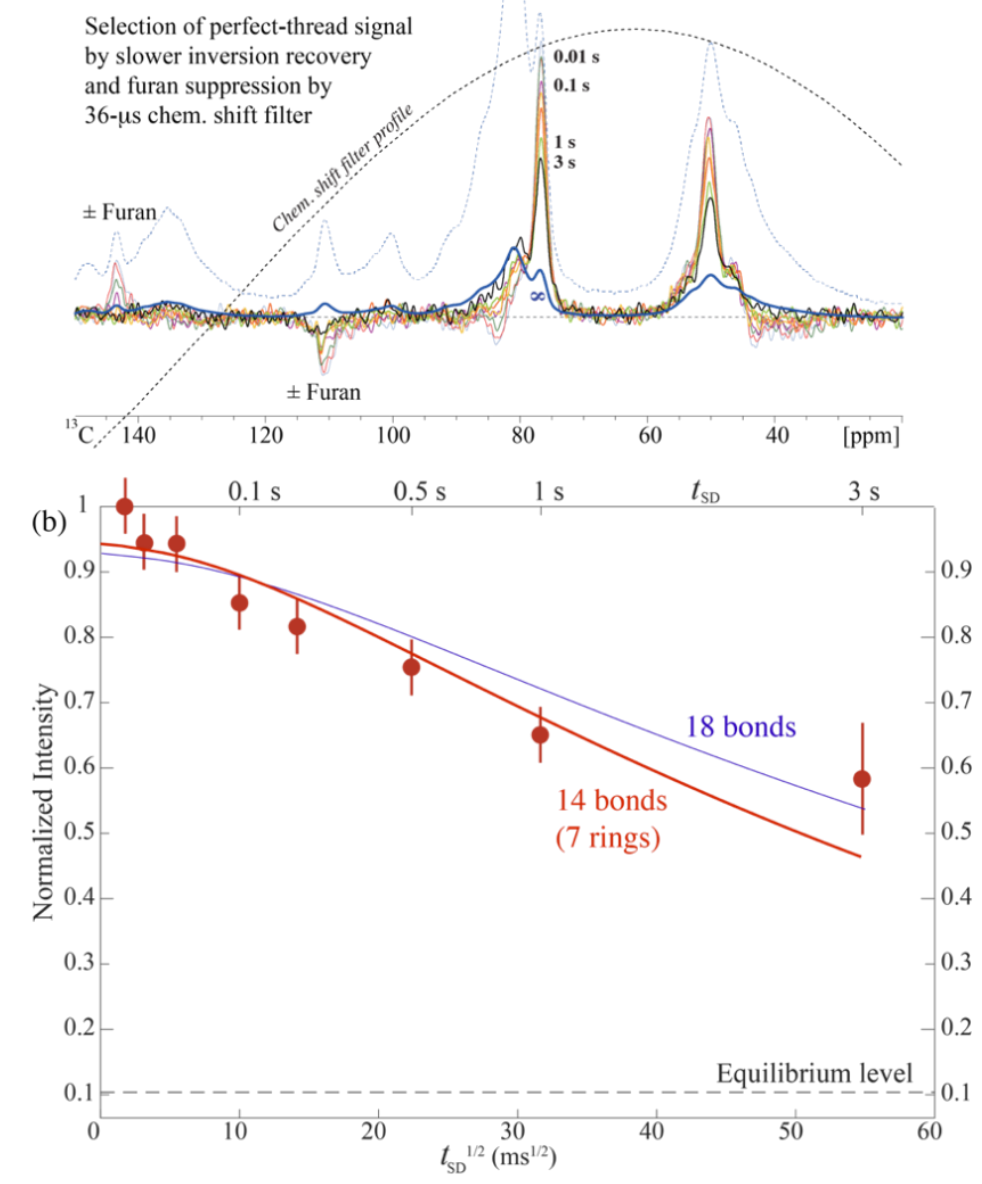

Figure 6. One-dimensional ${ }^{13} \mathrm{C}$ spin-exchange experiment after inversion recovery and chemicalshift filtering that selects the magnetization of perfect thread segments in ${ }^{13} \mathrm{C}$-furan-derived nanothreads. The pulse sequence is shown in Figure S7. (a) Series of spectra as a function of mixing time. The excitation profile of the 36-us chemical-shift filter aimed at suppressing the net magnetization of furan is shown by a dashed line. (b) Normalized intensity of the 77-ppm peak as a function of the square-root of the spin-exchange time. Thick red and thin blue lines: Fit curves for spin exchange after inversion recovery to the zero crossing of the matrix for perfect-thread segments that are 14 and 18 bonds in length, respectively.

The series of spectra in Figure 6a shows the expected decay of the sharp perfect-thread signals with increasing mixing time, towards long-time equilibrium spectrum (the regular spectrum scaled to the same total area), which is also shown, as a thick blue line. The intensity of the sharp 77-ppm 
peak is plotted as a function of the square-root of the mixing time in Figure 6b. Two results of full simulation of inversion recovery with relaxation and spin diffusion, followed by further spin diffusion out of the perfect thread segments as discussed below, see Figure S8c,d, are shown.

Quantum-chemical chemical shift prediction. The perfect threads show characteristic chemical shifts, in particular of the 77-ppm peak, which is quite well resolved from most of the other OCH signals. The fact that only two sharp peaks are observed is also structurally relevant. By comparison with chemical shifts from quantum-chemical simulations, one can rule out many structural models and identify the likely correct model. Figure 7 shows the structures of syn, anti, and syn/anti furan-derived nanothreads, marked with the corresponding computed chemical shifts of symmetry-distinct carbons. The syn/anti structure has four carbon sites with four distinct predicted chemical shifts of 79.7, 50.5, 83.7 and $54.4 \mathrm{ppm}$; this is clearly incompatible with the experimentally observed pair of peaks. The syn structure is computed to have chemical shifts of 87.2 and $49.0 \mathrm{ppm}$, deviating significantly from the observed 77 and $50 \mathrm{ppm}$. Only the computed peaks for anti threads, at 79.3 and $49.6 \mathrm{ppm}$, are in good agreement with experiment. Note also that prior analysis of X-ray diffraction data on furan nanothread packing geometries strongly favors anti over syn, as the narrower and more uniform cross-section of syn threads is not compatible with the experimentally observed Friedel spacings. ${ }^{22}$

In addition to the syn and anti structures formed by [4+2] reactions, a product of [2+2] reactions and a 1,3-polymer, ${ }^{73}$ see Figure 7, bottom, were also analyzed. Each contains two distinct $\mathrm{C}$ sites and eight distinct $\mathrm{C}-\mathrm{H}$ orientations, so they are compatible with the experimental NMR results. Identification of one of these structures would have major implications for the nanothread formation mechanism. In structures optimized at the DFTB level, the chemical shifts were 89.3 ppm and $51.2 \mathrm{ppm}$ for the [2+2] polymer, and 81.5 and $44.4 \mathrm{ppm}$ for the 1,3-polymer. These are clearly inconsistent with the experimental values of $77 \mathrm{ppm}$ and $50 \mathrm{ppm}$. To ensure that these discrepancies were not due to structural distortions, we also optimized the axial unit cell parameters for the two polymers at the DFT level. The chemical shifts changed only slightly, to $90.3 \mathrm{ppm}$ and $51.4 \mathrm{ppm}$ for the [2+2] polymer, and $82.1 \mathrm{ppm}$ and $44.7 \mathrm{ppm}$ for the 1,3-polymer. The discrepancy from the experimental values was not significantly reduced. 

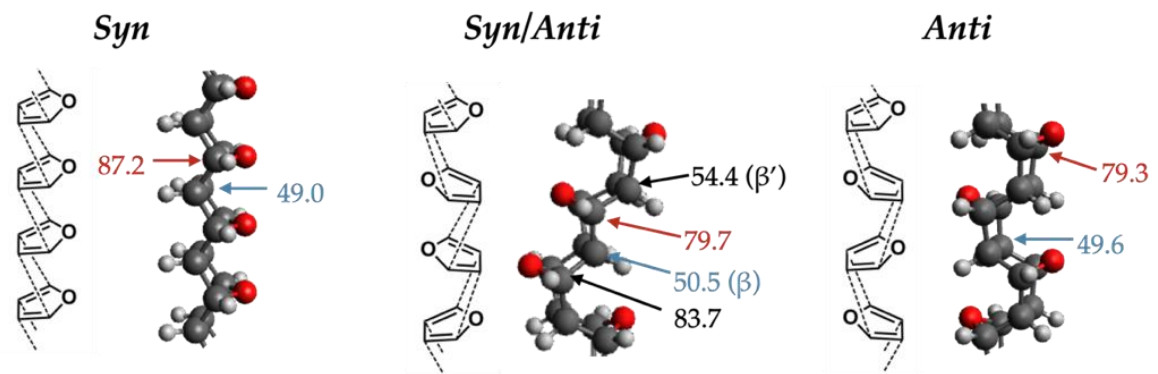

Experiment

C1: 77 ppm

C2: $50 \mathrm{ppm}$

[2+2] polymer

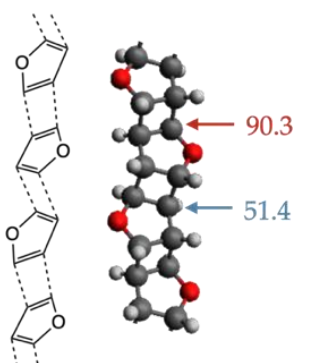

1,3-polymer

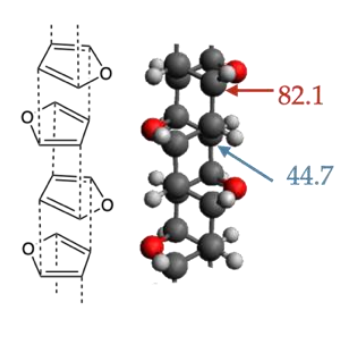

Figure 7. ${ }^{13} \mathrm{C}$ chemical shifts from quantum-chemical simulations in five types of furan-derived nanothreads, demonstrating the close agreement of only anti threads to the experimentally measured shifts.

Counting differently oriented $\mathrm{C}-\mathrm{H}$ bonds in perfect threads. Advanced solid-state ${ }^{13} \mathrm{C}$ NMR can also identify ordered ${ }^{13} \mathrm{C}$-enriched nanothreads based on their symmetry. Straight syn threads have the highest symmetry, with translation by one oxygen-oxygen distance along the thread axis leaving the structure unchanged; this means that there are only four differently oriented $\mathrm{C}$-H bonds in this structure if it is straight and not twisted. In anti threads, the structural period along the thread axis contains eight differently oriented $\mathrm{C}-\mathrm{H}$ bonds, and in syn/anti threads the corresponding number is 12 .

The number of differently oriented $\mathrm{C}-\mathrm{H}$ bonds (technically, magnetically inequivalent sites) can be determined by centerband-only detection of exchange (CODEX) ${ }^{13} \mathrm{C}$ NMR, ${ }^{37}$ taking advantage of ${ }^{13} \mathrm{C}-{ }^{13} \mathrm{C}$ spin exchange. CODEX measures the intensity of a stimulated echo of the recoupled chemical-shift anisotropy for each resolvable isotropic chemical shift position. In the full dephasing limit, with long enough recoupling time $N t_{r}$, the observed normalized intensity $S / S_{0}$ after the spin-exchange time is the fraction of magnetization in sites with the same $\mathrm{C}-\mathrm{H}$ orientation as before the spin-exchange time; this is the inverse of the number of magnetically inequivalent sites.

Figure 8 shows the normalized intensity $S / S_{0}$ for the 81 -ppm signal and for the $77-\mathrm{ppm}$ peak in perfect threads. The latter shows a faster initial decay, passing quickly through 0.25 , the final level 
for straight syn threads, and reach a slowly decreasing equilibrium value near $1 / 8$, the value for straight anti threads. Simulation of ${ }^{13} \mathrm{C}$ spin exchange in a perfect-thread "ladder" structure with a one-bond exchange rate constant of $k=80 \mathrm{~Hz}$ (see the SI for details) produced the bold orange curve for anti and the dash-dotted curve for syn threads. The former provides an excellent fit to the data up to $50 \mathrm{~ms}$. The additional slow signal decrease can be explained mostly by the finite length of the perfect thread segments: when magnetization diffuses out, see Figure S8e,f, there is a large change in chemical shift and corresponding loss of CODEX signal.

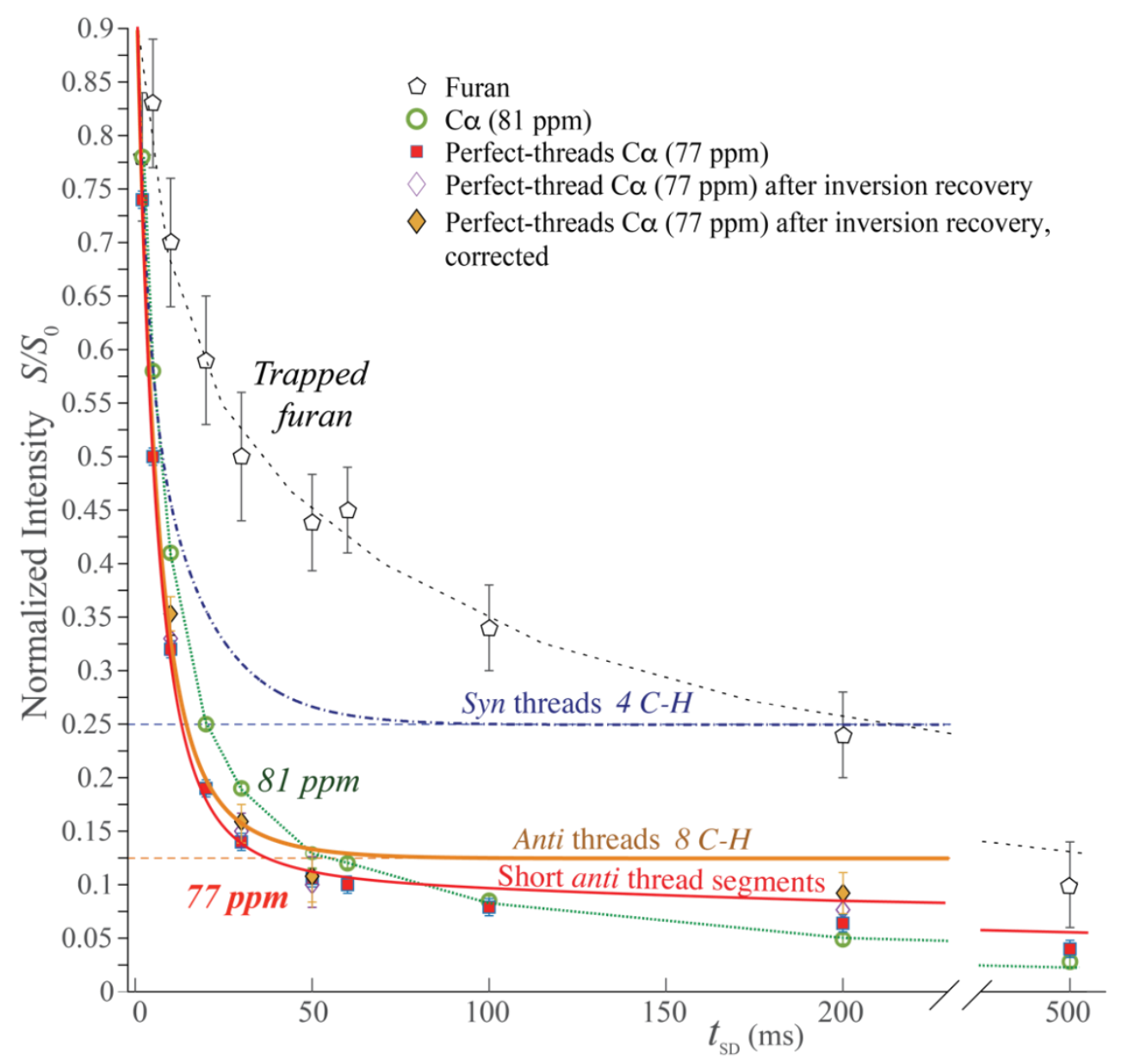

Figure 8. Counting the number of different $\mathrm{C}-\mathrm{H}$ bond orientations in ${ }^{13} \mathrm{C}$-furan-derived nanothreads by CODEX ${ }^{13} \mathrm{C}$ NMR. The plot shows the normalized CODEX signal intensity $S / S_{0}$ recorded at $81 \mathrm{ppm}$ (open green circles), $77 \mathrm{ppm}$ (filled red squares; perfect threads), and $143 \mathrm{ppm}$ (open pentagons; trapped furan). Filled diamonds: 77-ppm intensity after perfect-thread selection by inversion recovery correction for the isotropic-chemical-shift exchange quantified in Figure $6 \mathrm{~b}$. Horizontal lines at $1 / 4$ and $1 / 8$ intensity indicate the equilibrium signal levels in straight syn and anti threads, respectively, while full spin-exchange simulations yielded the dash-dotted blue and the thick orange curves, respectively. Solid red curve: Simulated CODEX decay for perfect anti thread segments that are 14 bonds in length. 
Comparison of the CODEX decays of OCH sites in perfect and defective threads in Figure 8 reveals distinctive differences. The perfect threads show a faster initial decay, which can be attributed to a more perfect "ladder" structure with two parallel ${ }^{13} \mathrm{C}^{13} \mathrm{C}$ couplings along the thread axis, while alkenes reduce the number of ${ }^{13} \mathrm{C}-{ }^{13} \mathrm{C}$ bonds in defective threads. At long times, the perfect threads show a slower decay than the matrix, indicating fewer differently oriented $\mathrm{C}-\mathrm{H}$ bonds.

Probing the size of perfect-thread domains. Since ${ }^{13} \mathrm{C}$ spin diffusion occurs primarily along the thread axis, it provides little information on the lateral size of perfect-thread clusters or domains. This information can be obtained instead from ${ }^{1} \mathrm{H}$ spin diffusion. Hydrogen atoms are at the periphery of a given nanothread and therefore relatively close to ${ }^{1} \mathrm{H}$ in neighboring threads, so ${ }^{1} \mathrm{H}$ spin diffusion between threads will be fairly fast. ${ }^{1} \mathrm{H}$ spin diffusion has been studied and exploited quite extensively in heterogeneous polymers, and the spin diffusion coefficient in rigid polymers is known to have a value of $D=0.8 \pm 0.2 \mathrm{~nm}^{2} / \mathrm{ms}^{72}$ This means that in domains of $10 \mathrm{~nm}$ diameter, magnetization equilibrates within about $100 \mathrm{~ms}$.

Figure S9 shows cross sections, along the ${ }^{13} \mathrm{C}$ dimension, from 2D ${ }^{1} \mathrm{H}-{ }^{13} \mathrm{C}$ HetCor spectra with ${ }^{1} \mathrm{H}$ spin diffusion during a mixing time after ${ }^{1} \mathrm{H}$ evolution and before the cross polarization to ${ }^{13} \mathrm{C}$. Solid lines are cross sections at a ${ }^{1} \mathrm{H}$ chemical shift of $6.2 \mathrm{ppm}$ (alkenes), while dashed lines were obtained at $2.2 \mathrm{ppm}\left(\mathrm{C}_{\beta}-\mathrm{H}\right)$ (scaled by 0.54 to generally match at the major 80 -ppm peak). Fast transfer from alkenes to alkyls generally, within $1 \mathrm{~ms}$, is followed by slower spin diffusion to the perfect threads with the peaks at 77 and $50 \mathrm{ppm}$, which reaches completion between 3 and $10 \mathrm{~ms}$. This slightly longer but still fairly short transfer time corresponds to perfect-thread 'domains' of $1-3 \mathrm{~nm}$ in diameter.

\section{DISCUSSION}

Alkenes. Two types of alkene-containing rings have been identified, based on distinctive cross peaks and diagonal peaks (or their absence): (i) Symmetric $C-C=C-C\left(C_{\beta}=C_{\beta}\right)$ alkenes are found in $\sim 24 \%$ of all $\mathrm{C}_{4} \mathrm{H}_{4} \mathrm{O}$ rings. This fully accounts for the reduced intensity of the alkyl $\beta$-carbon intensity and of the alkyl $\mathrm{C}_{\beta}-\mathrm{C}_{\beta}$ diagonal peak and suggests that these motifs dominate the nonalkyl portion of the thread backbone. Such a conclusion is further supported by the indication that the majority of these symmetric alkenes are part of a locally regular structure, with a relatively well-defined 81-ppm OCH signal and with intermolecular $\mathrm{C}_{\alpha}-\mathrm{C}_{\alpha}$ bonds, which account for some of the otherwise unexpected $\mathrm{C}_{\alpha}-\mathrm{C}_{\alpha}$ diagonal intensity in Figure 2a. A distinct minority of these rings occur as defects in a variety of different environments, with a wide range of $\mathrm{OCH}$ chemical 
shifts (79-90 ppm). (ii) Asymmetric $\mathrm{C}=\mathrm{C}-\mathrm{C}-\mathrm{C}\left(\mathrm{C}_{\alpha}=\mathrm{C}_{\beta}\right)$ alkenes are found in $\sim 12 \%$ of all rings. Their lack of an alkyl $\mathrm{C}_{\beta}-\mathrm{C}_{\beta}$ bond help explain the reduced alkyl $\mathrm{C}_{\beta}-\mathrm{C}_{\beta}$ diagonal peak in the sheared DQ/SQ NMR spectrum. The asymmetric alkenes are closely associated with alkyl threads, according to the fast ${ }^{13} \mathrm{C}$ spin exchange. Both alkene motifs can be incorporated into a thread backbone and may be associated with the initiation (asymmetric) or termination (symmetric) of nanothread polymerization via cycloaddition reactions, and/or polymerization pathways that propagate a single polymerization bond chain along the thread axis, potentially via radical polymerization pathway. ${ }^{73}$ The presence of alkene-containing rings along the thread backbone, particularly in a locally regular structure, suggests opportunities for further functionalization, solvation and intercalation, and may suggest thread structures with intermittent alkene "hinges" between alkyl "rods", depending on the degree of completion of the thread-forming reaction. The relative ratio of alkyl and alkene components is likely amenable to experimental control through tuning of reaction conditions.

Trapped furan. Two unusually sharp peaks of $=\mathrm{C}-\mathrm{H}$ moieties are observed at 111 and $142 \mathrm{ppm}$, close to the resonance positions of the two carbons in furan. They are directly bonded, according to cross peaks in Figure 2. The expected $111 \mathrm{ppm}$ diagonal peak of two chemically equivalent $\beta$ carbons is also observed in the sheared DQ/SQ spectrum of Figure 2a. Furthermore, the ${ }^{1} \mathrm{H}$ chemical shifts of 7.3 and $6.3 \mathrm{ppm}$, see Figure S3, match those in furan. Thus, it is clear that these signals, which correspond to $7 \%$ of all $\mathrm{C}$, arise from trapped monomer. Incomplete dipolar dephasing, see Figure 1d, indicates large-amplitude mobility. Still, cross polarization shows that the $\mathrm{C}-\mathrm{H}$ dipolar coupling has not been averaged to zero and these furan molecules are coupled quite strongly to the immobile matrix, receiving magnetization from it via ${ }^{1} \mathrm{H}$ spin diffusion within $1 \mathrm{~ms}$ and via ${ }^{13} \mathrm{C}$ spin exchange on the 0.5 -s time scale.

Initially, two distinct sharp peaks of isotropically mobile liquid- or gas-like furan were also observed in direct-polarization ${ }^{13} \mathrm{C}$ and ${ }^{1} \mathrm{H}$ spectra, see Figures $\mathrm{S} 10$ and $\mathrm{S} 11$. The signals disappeared after venting the sample (i. e. opening the cap of the rotor) for a few minutes and reappeared only to a small extent over the course of three weeks.

The mobile furan undergoes the fastest ${ }^{13} \mathrm{C}$ relaxation of all carbons in the sample (see Figure 5), which is consistent with larger-amplitude fluctuating fields with spectral density at the NMR Larmor frequency due to the significant mobility of the furan molecules. A gradual slow-down of ${ }^{13} \mathrm{C}$ relaxation of the nanothreads over the course of a few weeks suggests that it is driven significantly by ${ }^{13} \mathrm{C}$ spin diffusion from furan, whose concentration decreased with time, in particular due to venting. 
Perfect thread structure. The 2D and selective 1D NMR spectra in Figures $4 \mathrm{~b}$ and 5 show convincing evidence of the presence of $\sim 10 \%$ of fully saturated perfect threads. These exhibit two sharp peaks, at $77 \mathrm{ppm}$ of $\mathrm{OCH}$ and at $50 \mathrm{ppm}$ of $\mathrm{CH}$ not bonded to $\mathrm{O}$, in a 1:1 intensity ratio. The line width of $140 \mathrm{~Hz}$ to $200 \mathrm{~Hz}$ is spinning-speed dependent and mostly homogeneous in nature, according to $T_{2, \mathrm{C}}$ measurements. Most of the broadening can be attributed to two or three one-bond ${ }^{13} \mathrm{C}-{ }^{13} \mathrm{C}$ couplings, including two ${ }^{1} J_{\mathrm{C}-\mathrm{C}}$ couplings of $\sim 40 \mathrm{~Hz}$. The bonding in the perfect threads documented by ${ }^{13} \mathrm{C}_{-1}^{13} \mathrm{C}$ NMR involves multiple $\mathrm{C}_{\alpha}-\mathrm{C}_{\beta}$ bonds, a $\mathrm{C}_{\beta}-\mathrm{C}_{\beta}$ bond, but no $\mathrm{C}_{\alpha}-\mathrm{C}_{\alpha}$ bond. Threads of this kind, see Figure 7, have been predicted to form by [4+2] cycloaddition. ${ }^{22}$

The small number of peaks indicates a high symmetry of the perfect threads, higher than in syn/anti threads, which contain four distinct carbon sites. Ab initio calculations confirm that there is no accidental degeneracy of chemical shifts, so our data clearly rule out syn/anti threads. Pure syn or anti threads are both compatible with the number of signals observed. However, syn threads are excluded by several observations, while anti threads can explain all the data. CODEX NMR shows that the perfect threads have at least eight $\mathrm{C}-\mathrm{H}$ bond orientations, more than the four in syn threads. The OCH chemical shift in syn threads according to $a b$ initio calculations is $87 \mathrm{ppm}$, which deviates from the observed 77-ppm OCH signal by $10 \mathrm{ppm}$.

Slower $T_{1 \mathrm{C}}$ relaxation of the perfect threads can be attributed to reduced fluctuating magnetic fields with rate near the NMR Larmor frequency, due to smaller-amplitude motions of or near the perfect threads. The perfect threads are more rigid than the defective threads and/or less accessible to the mobile, fast-relaxing trapped furan.

${ }^{13} \mathrm{C}$ spin diffusion along the nanothreads. The average length of the perfect thread segments can be determined by analyzing the ${ }^{13} \mathrm{C}$ spin exchange with the surrounding imperfect segments, on the scale of several nanometers. ${ }^{13} \mathrm{C}$ spin exchange along a given thread can occur relatively fast by means of an uninterrupted sequence of strong $1.54-\AA$ one-bond couplings of about $2 \mathrm{kHz}$ in magnitude, leading to an average exchange rate of $k=80 \mathrm{~Hz}$ under magic-angle spinning conditions according to the CODEX decay constant. The couplings between carbons in different threads are much weaker, given the thread center-to-center distance of $6.5 \AA$. At an estimated 5.5 $\AA$ closest approach of carbons in neighboring threads, the coupling strength is only $0.04 \mathrm{kHz}, 23$ times weaker than within a thread. Truncation of the weak inter-thread couplings by the strong intra-thread ones with which their Hamiltonian does not commute further slows down interthread ${ }^{13} \mathrm{C}$ spin exchange. ${ }^{13} \mathrm{C}$ spin exchange or spin diffusion in nanothreads can therefore be well approximated as a one-dimensional process along a given thread.

After many one-bond spin-exchange steps, the process approaches spin diffusion. The ${ }^{13} \mathrm{C}$ spin diffusion coefficient along the thread axis can be calculated from the exchange rate $k$ and the step 
size $a$, which is the ${ }^{13} \mathrm{C}-{ }^{13} \mathrm{C}$ distance projected onto the thread axis, $D=k a^{2}=80 \mathrm{~Hz}(0.12 \mathrm{~nm})^{2}=$ $0.8 \mathrm{~nm}^{2} / \mathrm{s}$. If distances are reckoned in bond lengths, $D=80$ (bond length) ${ }^{2} / \mathrm{s}$. The magnetization remaining on the source location after spin diffusion for time $t$ is the local magnetization density $M(x, t)$ times the width of the region associated with the source spin (which is $a$ ) can be obtained from the normalized Gaussian point spread function of diffusion as $M(0, t) \times a=$ $a / \sqrt{22 D t}=0.5 / \sqrt{k t}$. For example, in furan nanothreads after $t=1 \mathrm{~s}, 3 \%$ of the magnetization remains at the source, or one could say that the magnetization has spread over $\sim 30$ bonds, which may correspond to 60 carbons due to the ladder structure of nanothreads. This is one good measure of the reach of ${ }^{13} \mathrm{C}$ spin diffusion and amenable to experiment, for instance as the decrease in the diagonal intensity in $2 \mathrm{D}$ exchange NMR. Alternatively, the range of $\pm \sigma$, with the root mean square displacement $=\sqrt{2 D t}$, can be considered. In terms of bonds, the range is $2 / a=2 \sqrt{k t}$, which gives a value of 18 bonds after $1 \mathrm{~s}$ of spin diffusion. This value is smaller than the previous estimate since the range of $\pm \sigma$ accounts for only $68 \%$ of the area of the Gaussian point spread function.

Determining the length of perfect thread segments. Differential ${ }^{13} \mathrm{C}$ inversion recovery shows that perfect and defective thread segments are not intimately mixed. Simulation of simultaneous relaxation and spin exchange, see Figure S8ab, show that regardless of the intrinsic relaxation times, such a large difference $(\sim 2 \mathrm{~s})$ in observed $T_{1 \mathrm{C}}$ is possible only if the perfect thread segment is at least 14 bonds in length. Considering that the $90 \%$ of defective threads are then $\sim 126$ bonds in length, this result is in agreement with the 18- to 30-bond length scale of ${ }^{13} \mathrm{C}$ spin diffusion along the thread on the second time scale estimated in the preceding paragraph. On the other hand, the relatively fast intensity decrease due to spin diffusion out of the perfect threads after the zero crossing of the matrix magnetization, see Figure 6, can only be fit if the perfect thread segment is at most 14 bonds in length. ${ }^{13} \mathrm{C}$ spin diffusion out of the perfect thread segment also explains most of the gradual decrease of the asymptote in the CODEX decay in Figure 8. The simulated time evolutions of the magnetization distributions in all these cases are documented in Figure S8.

Accordingly, fast ${ }^{1} \mathrm{H}$ spin diffusion (see Figure S9) also shows that the perfect threads are near defective threads and clearly do not form large crystals. On the other hand, some clustering of perfect threads is likely: even the random probability that one of the six neighbors of a perfect thread is also perfect exceeds $50 \%$. The ability of furan-derived nanothread samples to produce sharp quasi-six-fold X-ray diffraction ${ }^{22}$ suggests that the alkene portions of threads have geometrical characteristics sufficiently similar to the fully saturated alkyl components that they can pack into a common well-ordered lattice. 


\section{CONCLUSIONS}

${ }^{13} \mathrm{C}$-enrichment of furan by custom synthesis followed by modest-pressure synthesis of ${ }^{13} \mathrm{C}$ enriched nanothreads enabled a detailed characterization of the reaction products by a full complement of advanced solid-state NMR techniques, with validation by ab initio calculation of chemical shifts. The ${ }^{13} \mathrm{C}$ NMR spectrum was complex, with more than a dozen distinct features, but almost all (>95\%) represented $\mathrm{CH}$ moieties are as expected in nanothreads, with only $2-4 \%$ $\mathrm{CH}_{2}, 0.3 \% \mathrm{C}=\mathrm{O}$, and $0.3 \% \mathrm{COO}$ groups, according to spectral editing. Different components were quantified by integration of the fully equilibrated direct-polarization spectrum. The fraction of $\mathrm{sp}^{2}-$ hybridized $\mathrm{C}$ was $25 \%$, corresponding to $40 \%$ of $\mathrm{C}_{4} \mathrm{H}_{4} \mathrm{O}$ rings. Symmetric and asymmetric alkenecontaining rings as well as trapped furan were identified by ${ }^{13} \mathrm{C}-{ }^{13} \mathrm{C}$ and ${ }^{1} \mathrm{H}-{ }^{13} \mathrm{C}$ NMR. The most intriguing component observed was fully saturated perfect anti furan-derived nanothread segments, with two distinct, sharp peaks, accounting for ca. $10 \%$ of the material. The bonding patterns in these periodic structures deduced from DQ/SQ NMR was that of a [4+2] cycloaddition product. While the small number of chemically inequivalent carbon sites eliminated low-symmetry syn/anti threads, the large number of magnetically inequivalent ones (i.e., distinct $\mathrm{C}-\mathrm{H}$ orientations) in CODEX NMR was incompatible with the high-symmetry syn threads. Anti threads with two chemically and eight magnetically inequivalent sites provide the only consistent fit of the experimental data. These conclusions were convincingly corroborated by quantum-chemical simulations, which showed good agreement of isotropic chemical shifts only for the anti threads. This represents the first molecular-level identification of a specific type of nanothread. The typical length of the perfect, fully saturated thread segments was around 14 bonds and they accordingly constitute small clusters (according to ${ }^{13} \mathrm{C}$ and ${ }^{1} \mathrm{H}$ spin diffusion analyses) which likely reside within an overall hexagonal thread packing along with other, less-perfect or less-saturated brethren. The relatively slow $T_{1 \mathrm{C}}$ relaxation confirms the nanometer-scale length of the periodic perfect structure, indicates that the perfect threads are particularly rigid, and enables their selective observation in ${ }^{13} \mathrm{C} \mathrm{NMR}$.

\section{ASSOCIATED CONTENT}

\section{Supporting Information}

The Supporting Information will be available free of charge on the ACS Publications website. Additional NMR spectra, simulations of magnetization exchange, and details about the synthesis of ${ }^{13} \mathrm{C}_{4}$-furan.(PDF)

\section{AUTHOR INFORMATION}

\section{Corresponding Author}

*srohr@brandeis.edu 


\section{ACKNOWLEDGMENTS}

This work was funded by the Center for Nanothread Chemistry, a National Science Foundation (NSF) Center for Chemical Innovation (CHE-1832471). The solid-state NMR spectrometer used in this work was funded by the NSF MRI program (Award No. 1726346). 


\section{References}

1. Landa, S.; V., M., Sur l'adamantane, nouvel hydrocarbure extrait du naphte. Collection of Czechoslovak Chemical Communications 1933, 5, 1-5.

2. Prelog, V.; Rativoj, S., Über die Synthese des Adamantans. Berichte der deutschen chemischen Gesellschaft (A and B Series) 1941, 74 (10), 1644-1648.

3. Dresselhaus, M., Science of Fullerenes and Carbon Nanotubes. Elsevier: 1996; Vol. 35, p 965.

4. Kroto, H. W.; Heath, J. R.; O'Brien, S. C.; Curl, R. F.; Smalley, R. E., C60:

Buckminsterfullerene. 1985; Vol. 318, p 162--163.

5. Iijima, S., Helical microtubules of graphitic carbon. Nature 1991, 354 (6348), 56--58.

6. Charlier, J.-C.; Xavier, B.; Stephan, R., Electronic and transport properties of nanotubes. Reviews of Modern Physics 2007, 79 (2), 677--732.

7. Golder, M. R.; Ramesh, J., Syntheses of the Smallest Carbon Nanohoops and the Emergence of Unique Physical Phenomena. Accounts of Chemical Research 2015, 48 (3), 557--566.

8. Leonhardt, E. J.; Ramesh, J., Emerging applications of carbon nanohoops. Nature Reviews Chemistry 2019, 3 (12), 672--686.

9. Van Raden, J. M.; Leonhardt, E. J.; Zakharov, L. N.; Pérez-Guardiola, A.; Pérez-Jiménez, A. J.; Marshall, C. R.; Brozek, C. K.; Sancho-García, J. C.; Jasti, R., Precision Nanotube Mimics via Self-Assembly of Programmed Carbon Nanohoops. The Journal of Organic Chemistry 2020, 85 (1), 129-141.

10. Novoselov, K. S.; Geim, A. K.; Morozov, S. V.; Jiang, D.; Zhang, Y.; Dubonos, S. V.; Grigorieva, I. V.; Firsov, A. A., Electric field in atomically thin carbon films. Science 2004, 306 (5696), 666--669.

11. Sofo, J. O.; Chaudhari, A. S.; Barber, G. D., Graphane: A two-dimensional hydrocarbon. Physical Review B 2007, 75 (15), 153401.

12. Wen, X. D.; Hand, L.; Labet, V.; Yang, T.; Hoffmann, R.; Ashcroft, N. W.; Oganov, A. R.; Lyakhov, A. O., Graphane sheets and crystals under pressure. Proceedings of the National Academy of Sciences 2011, 108 (17), 6833--6837.

13. Strauss, M. J.; Asheghali, D.; Evans, A. M.; Li, R. L.; Chavez, A. D.; Sun, C.; Becker, M. L.; Dichtel, W. R., Cooperative Self-Assembly of Pyridine-2,6-Diimine-Linked Macrocycles into Mechanically Robust Nanotubes. Angewandte Chemie International Edition 2019, 58 (41), 14708--14714.

14. Vitaku, E.; Gannett, C. N.; Carpenter, K. L.; Shen, L.; Abrua, H. D.; Dichtel, W. R., Phenazine-Based Covalent Organic Framework Cathode Materials with High Energy and Power Densities. Journal of the American Chemical Society 2020, 142 (1), 16--20. 
15. Meng, Z.; Stolz, R. M.; Mirica, K. A., Two-Dimensional Chemiresistive Covalent Organic Framework with High Intrinsic Conductivity. Journal of the American Chemical Society 2019, 141 (30), 11929--11937.

16. Fitzgibbons, T. C.; Guthrie, M.; Xu, E.-S.; Crespi, V. H.; Davidowski, S. K.; Cody, G. D.; Alem, N.; Badding, J. V., Benzene-derived carbon nanothreads. Nature Materials 2015, 14 (1), 43-47.

17. Li, X.; Baldini, M.; Wang, T.; Chen, B.; Xu, E.-S.; Vermilyea, B.; Crespi, V. H.; Hoffmann, R.; Molaison, J. J.; Tulk, C. A.; Guthrie, M.; Sinogeikin, S.; Badding, J. V., Mechanochemical Synthesis of Carbon Nanothread Single Crystals. Journal of the American Chemical Society 2017, 139 (45), 16343-16349.

18. Li, X.; Wang, T.; Duan, P.; Baldini, M.; Huang, H.-T.; Chen, B.; Juhl, S. J.; Koeplinger, D.; Crespi, V. H.; Schmidt-Rohr, K.; Hoffmann, R.; Alem, N.; Guthrie, M.; Zhang, X.; Badding, J. V., Carbon Nitride Nanothread Crystals Derived from Pyridine. Journal of the American Chemical Society 2018, 140 (15), 4969-4972.

19. Fanetti, S.; Santoro, M.; Alabarse, F.; Enrico, B.; Bini, R., Modulating the H-bond strength by varying the temperature for the high pressure synthesis of nitrogen rich carbon nanothreads. Nanoscale 2020, 12 (8), 5233-5242.

20. Biswas, A.; Ward, M. D.; Wang, T.; Zhu, L.; Huang, H.-T.; Badding, J. V.; Crespi, V. H.; Strobel, T. A., Evidence for Orientational Order in Nanothreads Derived from Thiophene. The Journal of Physical Chemistry Letters 2019, 10 (22), 7164-7171.

21. Nobrega, M. M.; Teixeira-Neto, E.; Cairns, A. B.; Temperini, M. L. A.; Bini, R., Onedimensional diamondoid polyaniline-like nanothreads from compressed crystal aniline. Chemical Science 2018, 9 (1), 254-260.

22. Huss, S.; Wu, S.; Chen, B.; Wang, T.; Gerthoffer, M. C.; Ryan, D. J.; Smith, S. E.; Crespi, V. H.; Badding, J. V.; Elacqua, E., Scalable Synthesis of Crystalline OneDimensional Carbon Nanothreads through Modest-Pressure Polymerization of Furan. ACS Nano. 2021, 15 (3), 4134-4143.

23. Ward, M. D.; Tang, W. S.; Zhu, L.; Popov, D.; Cody, G. D.; Strobel, T. A., Controlled Single-Crystalline Polymerization of C10H8.C10F8 under Pressure. Macromolecules 2019, 52 (20), 7557-7563.

24. Gerthoffer, M. C.; Wu, S.; Chen, B.; Wang, T.; Huss, S.; Oburn, S. M.; Crespi, V. H.; Badding, J. V.; Elacqua, E., 'Sacrificial' supramolecular assembly and pressure-induced polymerization: toward sequence-defined functionalized nanothreads. Chemical Science 2020, 11 (42), 11419-11424.

25. Friedrich, A.; Collings, I. E.; Dziubek, K. F.; Fanetti, S.; Radacki, K.; Ruiz-Fuertes, J.; Pellicer-Porres, J.; Hanfland, M.; Sieh, D.; Bini, R.; Clark, S. J.; Marder, T. B., Pressure- 
Induced Polymerization of Polycyclic Arene-Perfluoroarene Cocrystals: Single Crystal Xray Diffraction Studies, Reaction Kinetics, and Design of Columnar Hydrofluorocarbons. Journal of the American Chemical Society 2020, 142 (44), 18907-18923.

26. Tang, W. S.; Strobel, T. A., Evidence for Functionalized Carbon Nanothreads from $\pi$ Stacked, para-Disubstituted Benzenes. The Journal of Physical Chemistry C 2020, 124 (45), 25062-25070.

27. Huang, H.-T.; Zhu, L.; Ward, M. D.; Wang, T.; Chen, B.; Chaloux, B. L.; Wang, Q.; Biswas, A.; Gray, J. L.; Kuei, B.; Cody, G. D.; Epshteyn, A.; Crespi, V. H.; Badding, J. V.; Strobel, T. A., Nanoarchitecture through Strained Molecules: Cubane-Derived Scaffolds and the Smallest Carbon Nanothreads. Journal of the American Chemical Society 2020, 142 (42), 17944-17955.

28. Roman, R. E.; Kwan, K.; Cranford, S. W., Mechanical Properties and Defect Sensitivity of Diamond Nanothreads. Nano Letters 2015, 15 (3), 1585--1590.

29. Silveira, J. F. R. V.; Muniz, A. R., First-principles calculation of the mechanical properties of diamond nanothreads. Carbon 2017, 113, 260--265.

30. Zhan, H.; Zhang, G.; Tan, V. B. C.; Cheng, Y.; Bell, J. M.; Zhang, Y.-W.; Gu, Y., From brittle to ductile: a structure dependent ductility of diamond nanothread. Nanoscale 2016, 8 (21), 11177--11184.

31. Chen, M.-M.; Xiao, J.; Cao, C.; Zhang, D.; Cui, L.-L.; Xu, X.-M.; Long, M.-Q., Theoretical prediction electronic properties of Group-IV diamond nanothreads. AIP Advances 2018, 8 (7), 075107.

32. Wang, Y.; Dong, X.; Tang, X.; Zheng, H.; Li, K.; Lin, X.; Fang, L.; Sun, G.; Chen, X.; Xie, L.; Bull, C. L.; Funnell, N. P.; Hattori, T.; Sano-Furukawa, A.; Chen, J.; Hensley, D. K.; Cody, G. D.; Ren, Y.; Lee, H. H.; Mao, H. K., Pressure-Induced Diels-Alder Reactions in C6 H6 -C6 F6 Cocrystal towards Graphane Structure. Angew. Chem. Int. Ed. Engl. 2019, 58 (5), 1468-1473.

33. Duan, P.; Li, X.; Juhl, S. J.; Chen, B.; Koeplinger, D.; Crespi, V. H.; Badding, J. V.; Schmidt-Rohr, K., The Chemical Structure of Carbon Nanothreads Analyzed by Advanced Solid-State NMR. J. Am. Chem. Soc. 2018, 140, 7658-7666

34. Wang, T.; Duan, P.; Xu, E.; Vermilyea, B.; Chen, B.; Li, X.; Badding, J. V.; SchmidtRohr, K.; Crespi, V. H., Constraining Carbon Nanothread Structures with Experimental and Calculated Nuclear Magnetic Resonance Spectra. Nano Lett. 2018, 4934-4942

35. Schmidt-Rohr, K.; Mao, J. D., Efficient CH-Group Selection and Identification in ${ }^{13} \mathrm{C}$ SolidState NMR by Dipolar DEPT and ${ }^{1} \mathrm{H}$ Chemical-Shift Filtering. J. Am. Chem. Soc. 2002, 124 (46), 13938-13948. 
36. Chen, Q.; Schmidt-Rohr, K., Backbone dynamics of the Nafion Ionomer Studied by ${ }^{19} \mathrm{~F}-{ }^{13} \mathrm{C}$ Solid-State NMR. Macromol. Chem. Phys. 2007, 208, 2189-2203.

37. deAzevedo, E. R.; Hu, W. G.; Bonagamba, T. J.; Schmidt-Rohr, K., Centerband-Only Detection of Exchange: Efficient Analysis of Dynamics in Solids by NMR. J. Am. Chem. Soc. 1999, 121 (36), 8411-8412.

38. Vu, C. C.; A., P. L., Synthesis of 13C4 furan. J. Labelled Compd. Radiopharm. 2005, 48 (2), 117--121.

39. Oger, C.; Laurence, B.; Thierry, D.; Marie, G. J., Are alkyne reductions chemo-, regio-, and stereoselective enough to provide pure (Z)-olefins in polyfunctionalized bioactive molecules? 2013; Vol. 113, pp 1313--1350.

40. Kraus, G. A.; Xuemei, W., An improved synthesis of 3-substituted furans from substituted butene- 1,4-diols. Synth. Commun. 1998, 28 (6), 1093--1096.

41. Qiu, J. C.; Pradhan, P. P.; Blanck, N. B.; Bobbitt, J. M.; Bailey, W. F., Selective oxoammonium salt oxidations of alcohols to aldehydes and aldehydes to carboxylic acids. Org. Lett. 2012, 14 (1), 350--353.

42. Zhdankin, V. V.; Stang, P. J., Recent Developments in the Chemistry of Polyvalent Iodine Compounds. 2002.

43. Fang, J.; Bull, C. L.; Loveday, J. S.; Nelmes, R. J.; Kamenev, K. V., Strength analysis and optimisation of double-toroidal anvils for high-pressure research. Rev. Sci. Instrum. 2012, 83 (9), 093902.

44. Klotz, S., Techniques in high pressure neutron scattering. CRC Press: 2012; p 1--255.

45. Bennett, A. E.; Rienstra, C. M.; Auger, M.; Lakshmi, K. V.; Griffin, R. G., Heteronuclear Decoupling in Rotating Solids. J Chem Phys 1995, 103 (16), 6951-6958.

46. Hahn, E. L., Spin Echoes. Phys. Rev. 1950, 80 (4), 580-594.

47. Dixon, W. T.; Schaefer, J.; Sefcik, M. D.; Stejskal, E. O.; McKay, R. A., Total suppression of sidebands in CPMAS C-13 NMR. J. Magn. Reson. (1969) 1982, 49 (2), 341345.

48. Fung, B. M.; Khitrin, A. K.; Ermolaev, K., An improved broadband decoupling sequence for liquid crystals and solids. J. Magn. Reson. 2000, 142 (1), 97-101.

49. Chen, Q.; Schmidt-Rohr, K., A Simple Scheme for Probehead Background Suppression in One-Pulse ${ }^{1} \mathrm{H}$ NMR. Solid State NMR 2004, 26, 11-14.

50. Mao, J. D.; Schmidt-Rohr, K., Methylene spectral editing in solid-state ${ }^{13} \mathrm{C}$ NMR by threespin coherence selection. J. Magn. Reson. 2005, 176 (1), 1-6.

51. Duan, P.; Schmidt-Rohr, K., Quick, selective NMR spectra of C-OH moieties in ${ }^{13} \mathrm{C}$ enriched solids. J. Magn. Reson. 2019, 301, 80-84.

52. Abragam, A., The Principles of Nuclear Magnetism. Oxford University Press: 1983; p 64. 
53. Hohwy, M.; Rienstra, C. M.; Jaroniec, C. P.; Griffin, R. G., Fivefold symmetric homonuclear dipolar recoupling in rotating solids: Application to double quantum spectroscopy. J Chem Phys 1999, 110 (16), 7983-7992.

54. Schmidt-Rohr, K., Complete Dipolar Decoupling of $13 \mathrm{C}$ and Its Use in Two-Dimensional Double-Quantum Solid-State NMR for Determining Polymer Conformations. J Magn Reson 1998, 131 (2), 209-17.

55. Caravatti, P.; Braunschweiler, L.; Ernst, R. R., Heteronuclear correlation spectroscopy in rotating solids. Chem. Phys. Lett. 1983, 100 (4), 305-310.

56. Bielecki, A.; Kolbert, A. C.; De Groot, H. J. M.; Griffin, R. G.; Levitt, M. H., FrequencySwitched Lee-Goldburg Sequences in Solids. In Advances in Magnetic Resonance, Elsevier: 1990; pp 111-124.

57. ACD/Structure Elucidator, version 12.01. Advanced chemistry development, Inc.: Toronto, ON, Canada, 2014.

58. Takegoshi, K.; Nakamura, S.; Terao, T., 13C-1H dipolar-assisted rotational resonance in magic-angle spinning NMR. Chemical Physics Letters 2001, 344 (5-6), 631-637.

59. Pickard, C. J.; Francesco, M., All-electron magnetic response with pseudopotentials: NMR chemical shifts. Phys. Rev. B: Condens. Matter 2001, 63 (24), 245101-245113.

60. Yates, J. R.; J., P. C.; Francesco, M., Calculation of NMR chemical shifts for extended systems using ultrasoft pseudopotentials. Phys. Rev. B: Condens. Matter 2007, 76 (2), 024401-024411.

61. Joyce, S. A.; R., Y. J.; J., P. C.; Francesco, M., A first principles theory of nuclear magnetic resonance J-coupling in solid-state systems. J Chem Phys 2007, 127 (20), 204107204110.

62. Green, T. F. G.; R., Y. J., Relativistic nuclear magnetic resonance J-coupling with ultrasoft pseudopotentials and the zeroth-order regular approximation. J Chem Phys 2014, 140 (23), 234106-234117.

63. Giannozzi, P.; Stefano, B.; Nicola, B.; Matteo, C.; Roberto, C.; Carlo, C.; Davide, C.; L., C. G.; Matteo, C.; Ismaila, D.; Dal Corso, A.; de; Gironcoli, S.; Fabris, S.; Fratesi, G.; Gebauer, R.; Gerstmann, U.; Gougoussis; C.; Kokalj, A.; Lazzeri, M.; MartinSamos, L.; Marzari, N.; Mauri, F.; Mazzarello, R.; Paolini, S.; Pasquarello, A.; Paulatto, L.; Sbraccia, C.; Scandolo; S.; Sclauzero, G.; Seitsonen, A. P.; Smogunov, A.; Umari, P.; Wentzcovitch, R. M., QUANTUM ESPRESSO: a modular and open-source software project for quantum simulations of materials. J. Phys.: Condens. Matter $\quad$ 2009, 21 (39), 395502-395520.

64. Ceresoli, D. Pseudopotentials. https://github.com/dceresoli/qe-gipaw/tree/master/pseudo (accessed December 17). 
65. Perdew, J. P.; Kieron, B.; Matthias, E., Generalized Gradient Approximation Made Simple. Phys. Rev. Lett. 1996, 77 (18), 3865-3868.

66. Charpentier, T., The PAW/GIPAW approach for computing NMR parameters: A new dimension added to NMR study of solids. Solid State Nucl. Magn. Reson. 2011, 40 (1), 120.

67. Ashbrook, S. E.; David, M., Combining solid-state NMR spectroscopy with first-principles calculations - a guide to NMR crystallography. Chem. Commun. 2016, 52 (45), 7186-7204.

68. Wang, T.; Duan, P.; Xu, E.-S.; Vermilyea, B.; Chen, B.; Li, X.; Badding, J. V.; Schmidt-Rohr, K.; Crespi, V. H., Constraining Carbon Nanothread Structures by Experimental and Calculated Nuclear Magnetic Resonance Spectra. Nano Lett. 2018, 18 (8), 4934-4942.

69. Ceppatelli, M.; Santoro, M.; Bini, R.; Schettino, V., High pressure reactivity of solid furan probed by infrared and Raman spectroscopy. JChem Phys 2003, 118 (3), 1499-1506.

70. Johnson, R. L.; Anderson, J. M.; Shanks, B. H.; Fang, X.; Hong, M.; Schmidt-Rohr, K., Spectrally edited 2D 13C-13C NMR spectra without diagonal ridge for characterizing ${ }^{13} \mathrm{C}$ enriched low-temperature carbon materials. J Magn Reson 2013, 234, 112-24.

71. Schmidt-Rohr, K.; Spiess, H. W., Multidimensional Solid-State NMR and Polymers. Academic Press: 1994.

72. Clauss, J.; Schmidt-Rohr, K.; Spiess, H. W., Determination of Domain Sizes in Polymers. Acta Polymer. 1993, 44, 1-17.

73. Hoffmann, R.; Crespi, V.; Chen, B., Theoretical Studies of Furan and Thiophene Nanothreads: Structures, Cycloaddition Barriers and Activation Volumes. ChemRxiv 2021, https://doi.org/10.26434/chemrxiv.14347163.v1. 